\title{
Suspensions of prolate spheroids in Stokes flow. Part 2. Statistically homogeneous dispersions
}

\author{
By IVAN L. CLAEYS $\dagger$ AND JOHN F. BRADY \\ Division of Chemistry and Chemical Engineering, California Institute of Technology, Pasadena, \\ CA 91125 , USA
}

(Received 14 August 1991 and in revised form 18 December 1992)

The simulation method for prolate spheroids in Stokes flow introduced in a companion paper (Claeys \& Brady 1993a) is extended to handle statistically homogeneous unbounded dispersions. The convergence difficulties associated with the slow decay of velocity disturbances at zero Reynolds number are overcome by applying O'Brien's renormalization procedure. The Ewald summation technique is employed to accelerate the evaluation of all mobility interactions. As a first application of this new method, the hydrodynamic transport properties of equilibrium hard-ellipsoid structures are calculated for aspect ratios ranging from 3 to 50. Calculated viscosities in the isotropic phase agree reasonably well with published experimental measurements.

\section{Introduction}

Fluid-particle systems in which the solid phase consists of elongated objects occur in all areas of science and engineering. Suspensions of rods are essential intermediates in many production processes, ranging from the fabrication of fibre-reinforced composites to the manufacture of paper from pulp. The permeability of filters and membranes depends on, apart from specific physico-chemical factors, the pressure drop across a fixed bed of fibres. The electrophoretic mobility of biopolymers in a gel is greatly influenced by steric and viscous interactions between the macromolecule and the filamentous network through which it moves. The molecular constituents of all liquid crystals are elongated, and self-diffusion in those anisotropic fluids is of great interest from both a practical and scientific standpoint. In all cases, from a rheologist's point of view, the physical system consists of a fluid in which non-spherical particles are suspended. In this article, we propose to calculate the hydrodynamic transport properties of such dispersions by extending the ideas set forth in our previous paper (Claeys \& Brady 1993a) to systems consisting of infinitely many particles. The approach is akin in concept to the simulation technique for unbounded suspensions of spheres known as 'Stokesian dynamics' (Brady \& Bossis 1988; Brady et al. 1988).

Rod dispersions are of particular interest because they can display radically different transport properties than the solvent, even at concentrations of solid matter so low that most other characteristics of the sample are unaltered. In the semi-dilute concentration regime especially (defined as the range of volume fractions $\phi$ such that $r_{p}^{-2}<\phi \leqslant r_{p}^{-1}$, with $r_{p}$ a characteristic length-to-breadth ratio for the particles), hydrodynamic interactions between the rods totally dominate the rheological response of the suspension, even if only minute quantities of fibres are present $(\phi \ll 1)$. The incommensurate increase of the extensional viscosity of fluids upon addition of minuscule amounts of dispersed fibrous material (Mewis \& Metzner 1974; Pittman \&

$\dagger$ Present address: Solvay Research \& Technology, rue de Ransbeek 310, 1120 Brussels, Belgium. 
Bayram 1990) is the most vivid illustration thereof. This dramatic enhancement of the resistance to stretch is typical for rod suspensions, and is not observed when the particles are spherical or disk-like. It is of tremendous relevance for the processing of fibre dispersions, and is the hydrodynamic analogue of the large directional increase in heat transport or electrical conductivity which can be achieved in fibre composites. The origin of these phenomena is now well understood (Batchelor 1971), and can be traced back to the observation that rods, which transmit energy, current or stress very effectively, influence the transport of these quantities over regions much larger than their own volume. In fact, while the concentration of the fibres scales with the length $L$ of the particles (for a given cross-sectional area), the domain over which their presence is felt grows with the cube of the length (Batchelor 1971; Shaqfeh \& Fredrickson 1990). Long, slender particles therefore affect the macroscopic properties far more than would be expected from their concentration, and increases of the Trouton viscosity by orders of magnitude can be attained by adding just fractions of a percent of rods to the suspension (provided the rods are slender enough, so that $\phi r_{p}^{2}=O(1)$, i.e. the suspension is semi-dilute).

Although the fundamental elements determining the hydrodynamic behaviour of rod suspensions are beginning to be understood, most analytical theories on fibre dispersions are asymptotic, and cease to be accurate at moderate concentrations or for small aspect ratios. In the dilute regime, a series expansion in $1 / \ln \left(r_{p}\right)$ is commonly used, so that first-order corrections can only be neglected in principle for extremely slender objects. Few materials, however, consist of straight rigid fibres of very high aspect ratio; slurries commonly contain particles which are distinctly non-spherical, but they usually resemble short rods rather than needles. Similarly, the shape of many proteins is well approximated by ellipsoids, but their length-to-width ratio seldom exceeds five or six. In the case of semi-dilute suspensions, the transport properties scale with the inverse of the logarithm of the volume fraction $\left(1 / \ln \left(\phi^{-1}\right)\right)$, and the aspect ratio ceases to matter. However, the simultaneous requirements $\phi r_{p}^{2}>1$ and $\phi \ll r_{p}^{-1}$ can hardly be met for slightly elongated particles, putting in doubt the applicability of these power series solutions for suspensions of short and thick rods. Our simulation method on the other hand accurately describes the rheology of such suspensions up to concentrations well beyond the dilute regime.

The model system we consider consists of prolate ellipsoids of revolution suspended in a Newtonian fluid. This class of particle shapes includes the sphere at one end of the spectrum and the line segment (i.e. an infinitely slender rod) at the other. The method can accommodate heterogeneous populations of spheroids, accepting polydispersity in both size and aspect ratio. The Reynolds number, based on the largest particle dimension, is assumed to be vanishingly small, so that the dynamics of the fluid phase are adequately described by the quasi-stationary creeping flow equations:

$$
\begin{gathered}
\nabla p=\mu \nabla^{2} \boldsymbol{u}, \\
\nabla \cdot \boldsymbol{u}=\mathbf{0} .
\end{gathered}
$$

The vector field $u$ represents the fluid velocity, $p$ is the associated pressure and $\mu$ the viscosity of the liquid in the absence of particles. The adoption of the Stokes equations (1.1) implies that inertial effects are negligible compared to viscous retardation on the lengthscale of the particles. The spheroids are, however, large enough to neglect the effects of Brownian motion. No-slip boundary conditions hold at the surfaces $A_{p}$ of the particles, which are considered to be undeformable:

$$
u(x)=U_{p}+\left(x-x_{p}\right) \wedge \Omega_{p} \text { for } x \in A_{p}
$$


The translational velocity $U_{p}$ is evaluated at the geometric centre $\boldsymbol{x}_{p}$ of the spheroid; $\boldsymbol{\Omega}_{p}$ represents the rotation rate of the particle.

Unbounded, statistically homogeneous suspensions are modelled by periodically replicating a suitable number $(N)$ of spheroids in space. Denoting the fluid phase by $V$ and its boundary by $\partial V$ ( $=$ the union of all the particle surfaces $A_{p}$ ), we thus require, for all sets $z$ of three integer coordinates, that

$$
\boldsymbol{x} \in \partial V \Leftrightarrow \boldsymbol{x}+\mathbf{x}_{\{z\}} \in \partial V \text { with } \mathbf{x}_{\{z\}}=\boldsymbol{B} \cdot \boldsymbol{z} .
$$

The columns of the $3 \times 3$ constant matrix $\boldsymbol{B}$ contain the basis vectors $\boldsymbol{b}_{i}(i=1,2,3)$ used to reproduce the $N$ references spheroids periodically in space. The ensemble $\mathscr{L}$ of all possible points $\mathbf{x}_{\{z\}}$ defines a lattice. In essence, (1.3) states that the microstructure is invariant with respect to translations along any of the basis vectors $b_{i}$ of the lattice $\mathscr{L}$. The only restriction placed on the basis vectors is that they should be compatible with the mutual impenetrability of the particles. They should, of course, also be linearly independent and form a complete basis set for the three-dimensional Euclidean space.

The simulation method outlined hereafter is general, and can be applied to track the particle trajectories, as illustrated for a finite number of spheroids in a previous paper (Claeys \& Brady 1993 a). In this article, however, we shall only present results in which the time evolution of the microstructure under the influence of the flow is not followed. Hence we shall not be concerned with the compatibility of the lattice $\mathscr{L}$ with any imposed deformation. $\dagger$ Instead, the hydrodynamic transport properties are computed for a few chosen, representative configurations. As we shall explain in $\$ 4$ (see also Brady \& Bossis 1988), the sedimentation rates, self-diffusivities, hindered diffusion coefficients, viscosity and permeability tensors can all be calculated simultaneously by Stokesian dynamics. These transport properties are reported in $\$ 6$ for equilibrium hard-ellipsoid structures generated by a Monte-Carlo procedure, and compared to available experimental data. Quite interestingly, we tracked the concentration dependence of several properties across the thermodynamic isotropic-to-nematic boundary. As is to be expected from a first-order phase transition, a discontinuous jump occurs at that point. In an effort to understand the hydrodynamics of these concentrated liquid crystalline dispersions, we also examined the transport properties of very regular structures of parallel spheroids. The findings of this model study are discussed in a separate publication (Claeys \& Brady 1993b).

The method proposed here is essentially a multipole moment technique for Stokes flow (Weinbaum, Ganatos \& Yan 1990). The basic ideas have already been laid down elsewhere, and the present work mostly concerns the adaptations needed for suspensions of infinite extent. It is now well documented (Batchelor 1972; Brady et al. 1988) that the slow decay of velocity disturbances in creeping flow lead to severely nonconvergent expressions for many transport properties in an infinite suspension, unless the interactions are properly renormalized and the appropriate 'backflow' integrals are subtracted from the results obtained by a simple-minded superposition of effects. In the next section, we shall discuss the application of O'Brien's (1979) method to correct these aphysical convergence problems. We shall then give a brief overview of the methodology of Stokesian dynamics for prolate spheroids (but refer to Claeys \& Brady $1993 a$ or Claeys 1991 for details), and discuss the application of the Ewald summation technique (Ewald 1921; Beenakker 1986) to accelerate the convergence of the calculations. Section 5 validates the method by comparing results obtained for spheroids to calculations on strings of beads. The overall agreement of both sets of

$\dagger$ The issue of kinematic compatibility is discussed by Zuzovsky, Adler \& Brenner (1983) for planar flows, and by Adler (1985) and Claeys (1991) for arbitrary straining fields. 
data demonstrates that the truncated multipole moment expansion technique which we use captures the essential hydrodynamics of 'rod' dispersions. Simulation results on the hydrodynamic transport properties of equilibrium hard-ellipsoid dispersions are discussed in $\S 6$. We close in $\S 7$ with a few comments about present and future work.

\section{A convergent formulation of hydrodynamic interactions in unbounded dispersions}

The Green's function for the governing equations (1.1) is called the Oseen tensor $J$ (Ladyzhenskaya 1963):

$$
J(x)=\frac{\delta}{r}+\frac{x x}{r^{3}},
$$

with $r=|x|$. According to (2.1), the velocity response at $x$ due to a point force $f$ at the origin, $u(x)=J \cdot f / 8 \pi \mu$, decays inversely proportionally with distance in an unbounded Newtonian fluid of viscosity $\mu$. Hence a simple-minded calculation of the sedimentation rate of an infinite collection of particles by superposing the contributions of all bodies (since each of them acts as a point force to a first approximation) diverges as $R_{\Gamma}^{2}$, with $R_{\Gamma}$ a characteristic size of the macroscopic system. The origin of these convergence difficulties is now well understood, and several procedures have been devised for overcoming them (Batchelor 1972; Batchelor \& Green 1972; Jeffrey 1973; Hinch 1977; O'Brien 1979). O'Brien's method is most conveniently adapted to microstructurally detailed numerical simulations, since it does not rely on preaveraging the interactions to obtain well-posed, absolutely convergent expressions for the transport properties. The application of the technique to Stokes flow has been documented earlier (Glendinning \& Russel 1982; Brady et al. 1988; Claeys 1991), and leads to the following expression for the fluid velocity $\boldsymbol{u}$ in a statistically homogeneous dispersion with number density $n_{\phi}$ :

$$
\begin{aligned}
\boldsymbol{u}(\boldsymbol{x})-\langle\boldsymbol{u}(\boldsymbol{x})\rangle & =\frac{2 n_{\phi}}{15 \mu}\left[2\left\langle\boldsymbol{Q}^{\prime}: \boldsymbol{\delta}\right\rangle-\left\langle\boldsymbol{\delta}: \boldsymbol{Q}^{\prime}\right\rangle\right]-\frac{1}{8 \pi \mu} \sum_{p=1}^{N} \int_{\boldsymbol{A}_{p}} \boldsymbol{J} \cdot \boldsymbol{\sigma} \cdot \mathrm{d} \boldsymbol{A}_{\boldsymbol{y}} \\
& -\frac{n_{\phi}}{8 \pi \mu} \int_{V-V_{q}}\left\{\langle\boldsymbol{F}\rangle \cdot \boldsymbol{J}+\langle\boldsymbol{T}\rangle \cdot \boldsymbol{R}+\langle\boldsymbol{S}\rangle: \boldsymbol{K}+\left\langle\boldsymbol{Q}^{\prime}\right\rangle \odot^{3} \cdot \boldsymbol{\nabla} \boldsymbol{\nabla}\right\} \mathrm{d} V_{\boldsymbol{y}}
\end{aligned}
$$

Equation (2.2) gives the fluid velocity $\boldsymbol{u}$ relative to the suspension average flow field $\langle\boldsymbol{u}\rangle$ at $x$ in terms of the stress tensor $\sigma=-p \delta+\mu\left(\nabla u+{ }^{\mathrm{T}} \nabla u\right)$ on the particles, and of the average force $\langle\boldsymbol{F}\rangle$, torque $\langle\boldsymbol{T}\rangle$, stresslet $\langle\boldsymbol{S}\rangle$ and quadrupole $\left\langle\boldsymbol{Q}^{\prime}\right\rangle$ exerted by the solid inclusions. The stresslet is defined as the symmetric part of the first moment of the force density on the particle surfaces, i.e.

$$
\boldsymbol{S}_{p}=-\int_{A_{p}} \frac{1}{2}\left[\boldsymbol{n} \cdot \boldsymbol{\sigma}\left\{\boldsymbol{y}-\boldsymbol{x}_{p}\right\}+\left\{y-x_{p}\right\} \sigma \cdot n\right]-\frac{1}{3} \delta\left[n \cdot \sigma \cdot\left(y-x_{p}\right)\right] \mathrm{d} A_{y},
$$

where the subscript $y$ on the elementary area $\mathrm{d} \boldsymbol{A}_{\boldsymbol{y}}=\boldsymbol{n} \mathrm{d} \boldsymbol{A}_{\boldsymbol{y}}$ indicates that the integration is carried out with respect to the position vector $y$; the normal $n$ points into the control volume $V$. The quadrupole $\boldsymbol{Q}_{p}^{\prime}$ is the second moment of the stress density of particle $p$ :

$$
Q_{p}^{\prime}=-\frac{1}{2} \int_{A_{p}} n \cdot \sigma\left[y-x_{p}\right]\left[y-x_{p}\right] \mathrm{d} A_{y}
$$


The summation in (2.2) includes the $N$ particles with centres enclosed in the volume $V$, which can be thought of as a large sphere of radius $R_{\Gamma}$ whose surface cuts indiscriminately through the solid and the liquid phases. The tensor $\boldsymbol{R}=\frac{1}{2} \varepsilon: \nabla J$ is the propagator for a point torque (or rotlet) in Stokes flow; $K=-3 x \boldsymbol{x x} / r^{5}$, and the notation $\odot^{m}$ stands for the $m$-fold nested contraction operator. We isolated the effect of the singularity at $x$ by surrounding it by an infinitesimal shell enclosing a volume of fluid $V_{\epsilon^{-}}$(This is strictly necessary only for the qaudrupolar contribution.) Physically, the first term in the volume integral represents the backflow of fluid, driven by the pressure gradient which balances the excess average force $\langle\boldsymbol{F}\rangle$ exerted by the particles (Brady et al. 1988). One can now let the dimensions $R_{\Gamma}$ of the control volume become arbitrarily large, because the 'backflow' generated in response to a non-vanishing average force, torque, stresslet or quadrupole cancels, in the mean, the cumulative effect of the solid particles on the fluid velocity. Hence the expression (2.2) for $u$ is absolute convergent.

We now derive the motion of the particles in the dispersion by means of Faxén laws. For ellipsoids, Brenner (1966) has shown that

$$
U_{p}=\frac{\sinh \mathrm{D}_{p}}{\mathrm{D}_{p}} u_{0}\left(x_{p}\right)
$$

with

$$
\mathrm{D}_{p}=\left(\mathrm{D}_{p}^{2}\right)^{\frac{1}{2}} \quad \text { and } \quad \mathrm{D}_{p}^{2}=\boldsymbol{A}_{p}: \nabla \nabla
$$

The symmetric positive definite second rank tensor $\boldsymbol{A}_{p}$ characterizes the surface $A_{p}$ of the ellipsoid: $\boldsymbol{x} \in A_{p} \Leftrightarrow \boldsymbol{A}_{p}^{-1}:\left(\boldsymbol{x}-\boldsymbol{x}_{p}\right)\left(\boldsymbol{x}-\boldsymbol{x}_{p}\right)=1$, with $\boldsymbol{x}_{p}$ the centre of the particle. The ambient fluid velocity $u_{0}$ includes any externally imposed flow as well as the disturbances due to all other particles in the suspension. Hence $u_{0}$ is given by (2.2), with the caveat that $p$ must be excluded from the sum. After a few algebraic manipulations (Claeys 1991), and accounting for the force $F_{p}$ acting on the particle, we obtain an absolutely convergent expression for the translational velocity $U_{p}$ of an ellipsoid in a statistically homogeneous unbounded suspension:

$$
\begin{aligned}
U_{p}-\left\langle u\left(x_{p}\right)\right\rangle= & \frac{2 n_{\phi}}{15 \mu}\left[2\langle\boldsymbol{Q}: \delta\rangle-\langle\delta: Q\rangle+\frac{1}{3}\left(\boldsymbol{A}_{p}: \delta\right)\langle\boldsymbol{F}\rangle-\frac{1}{6} \boldsymbol{A}_{p} \cdot\langle\boldsymbol{F}\rangle\right. \\
& \left.+\frac{1}{3}\langle(\boldsymbol{A}: \delta) \boldsymbol{F}\rangle-\frac{1}{6}\langle\boldsymbol{A} \cdot \boldsymbol{F}\rangle\right]+\boldsymbol{K}_{U F}^{p} \cdot \boldsymbol{F}_{p}-\frac{1}{8 \pi \mu} \sum_{q \neq p} \int_{A_{q}} \frac{\sinh \mathrm{D}_{p}}{\mathrm{D}_{p}} \boldsymbol{J} \cdot \boldsymbol{\sigma} \cdot \mathrm{d} \boldsymbol{A} \\
& -\frac{n_{\phi}}{8 \pi \mu} \int_{V-V_{c}}\left\{\left(1+\frac{1}{6} \mathrm{D}_{p}^{2}\right)\langle\boldsymbol{F}\rangle \cdot \boldsymbol{J}+\frac{1}{6}\left\langle\mathrm{D}^{2} \boldsymbol{F}\right\rangle \cdot \boldsymbol{J}\right. \\
& +\langle\boldsymbol{T}\rangle \cdot \boldsymbol{R}+\langle\boldsymbol{S}\rangle: \boldsymbol{K}+\langle\boldsymbol{Q}\rangle \odot^{3} \nabla \nabla \boldsymbol{J} \mathrm{d} V .
\end{aligned}
$$

Equation (2.5) is expressed in terms of the irreducible ellipsoidal quadrupole $\boldsymbol{Q}_{p}=\boldsymbol{Q}_{p}^{\prime}-\frac{1}{6} F_{p} \boldsymbol{A}_{p}$ (Claeys \& Brady $1993 a$; Claeys 1991). The dyadic $\boldsymbol{K}_{U F}^{p}$ relates the velocity of the spheroid to the force it exerts on the fluid, and is a well-known material constant (Brenner 1964). Expressions analogous to (2.5) can be obtained for the angular velocity relative to the vorticity of the imposed flow and the (zero) rate of deformation relative to the impressed rate of strain. A similar derivation is also possible for $\left.\nabla \nabla u\right|_{x_{p}}$ (the velocity counterpart of the quadrupole), but, as explained in Brady et al. (1988), this is only of marginal importance since all irreducible second moments are induced by particle interactions and are therefore of small amplitude. Expressions for higher multipoles are convergent even without applying O'Brien's method and do not need to be considered. 


\section{Methodology of Stokesian dynamics for unbounded suspensions of prolate spheroids}

The linearity of the Stokes equations guarantees the existence of a 'grand resistance tensor' $\mathscr{R}$ connecting the moments of the stress density on the particle surfaces (i.e. the force, torque, stresslet and higher multiples exerted by the spheroids) to their motion relative to the fluid. We chose to truncate the multipole expansion after the first two moments so that 11 unknowns are associated with each particle. These typically are the three components of the velocity, the three components of the rotation rate, and the five independent elements of the stresslet (a symmetric, traceless second-rank tensor, see $(2.3 a))$. Therefore, $\mathscr{R}$ is an $11 N \times 11 N$ tensor so that

$$
\left(\begin{array}{c}
\boldsymbol{F} \\
T \\
\boldsymbol{S}
\end{array}\right)=\mathscr{R} \otimes\left(\begin{array}{c}
U-\boldsymbol{U}_{\infty} \\
\boldsymbol{\Omega}-\boldsymbol{\Omega}_{\infty} \\
-\boldsymbol{E}_{\infty}
\end{array}\right)=\left(\begin{array}{lll}
\boldsymbol{R}_{F U} & \boldsymbol{R}_{F \Omega} & \boldsymbol{R}_{F E} \\
\boldsymbol{R}_{T U} & \boldsymbol{R}_{T \Omega} & \boldsymbol{R}_{T E} \\
\boldsymbol{R}_{S U} & \boldsymbol{R}_{S \Omega} & \boldsymbol{R}_{S E}
\end{array}\right) \otimes\left(\begin{array}{c}
U-U_{\infty} \\
\boldsymbol{\Omega}-\boldsymbol{\Omega}_{\infty} \\
-\boldsymbol{E}_{\infty}
\end{array}\right) .
$$

The operator $\otimes$ signifies the appropriate contraction. The vectors of length $3 N, \boldsymbol{U}, \boldsymbol{\Omega}$, $F$, and $T$, and the $3 N \times 3$ matrix $S$ group the translational and angular velocities of all $N$ particles, and the forces, torques and stresslets that they exert respectively. Similarly, the undisturbed ambient fluid velocity, vorticity and rate of strain at the geometric centres of the spheroids are lumped into $U_{\infty}, \boldsymbol{\Omega}_{\infty}$ and $\boldsymbol{E}_{\infty}$. Standard arguments in zeroReynolds-number hydrodynamics (Happel $\&$ Brenner 1973) show that $\mathscr{R}$ is positivedefinite and symmetric. More importantly, it is a purely geometric quantity, fully determined by the instantaneous configuration (and shape) of the particles. The greatest asset of Stokesian dynamics is its efficient and accurate algorithm to approximate this resistance tensor. The method was first developed for suspensions of spheres (Durlofsky, Brady \& Bossis 1987), and we showed in a preceding article (Claeys \& Brady 1993a) how it can be applied to a finite number of prolate spheroids. We now extend the approach to unbounded dispersions.

The first step in the procedure consists in forming the grand mobility tensor $M=\mathscr{R}^{-1}$ :

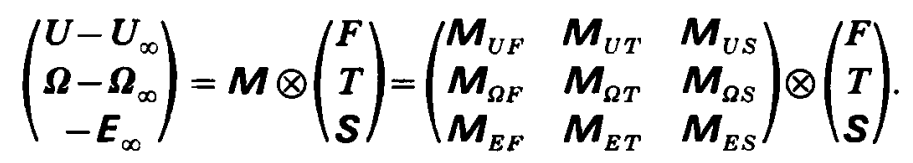

For a finite number of spheroids (Claeys \& Brady 1993a), $M$ is constructed by combining the singularity representation for the disturbance velocity (Chwang \& Wu $1974,1975)$ with the non-local formulation of the Faxén laws derived from the work of Kim (1985). The $\boldsymbol{M}_{U F}^{p q}$-coupling, for instance, which links the translational velocity of particle $p$ to the force exerted by $q$, can be written as

$$
\begin{aligned}
M_{U F}^{p q}=\frac{1}{32 \pi \mu c_{p} c_{q}} \int_{-c_{p}}^{c_{p}}\{1 & \left.+\left(c_{p}^{2}-\xi_{p}^{2}\right) \frac{1-e_{p}^{2}}{4 e_{p}^{2}} \nabla_{p}^{2}\right\} \\
& \times \int_{-c_{q}}^{c_{q}}\left\{1+\left(c_{q}^{2}-\xi_{p}^{2}\right) \frac{1-e_{q}^{2}}{4 e_{q}^{2}} \nabla_{q}^{2}\right\} J\left(\xi_{p}-\xi_{q}\right) \mathrm{d} \xi_{q} \mathrm{~d} \xi_{p}
\end{aligned}
$$

The distance $c_{q}$ from the centre to the foci of the generating ellipse, and the eccentricity $e_{q}$, defined as $c_{q} / a_{q}=\left[\left(1-b_{q}^{2} / a_{q}^{2}\right)\right]^{\frac{1}{2}}$, with $a_{q}$ and $b_{q}$ the lengths of the major and minor semi-axes respectively, characterize the dimensions of the spheroids. The vector $\xi_{q}=x_{q}+\xi_{q} d_{q}$, with $d_{q}$ the unit vector along the symmetry axis of the particle. One sees from (3.3a) that, as far as $M_{U F}$ is concerned, each spheroid is equivalent to a line of Stokeslets with uniform density, supplemented by potential dipoles $\nabla^{2} J$ distributed 
between the foci according to a parabolic density profile. The entire grand mobility tensor $M$ is built up of similar double integrals (Claeys \& Brady 1993a). As an alternative to $(3.3 a)$, a symbolic operator representation for the mobility elements can be used to get

$$
M_{U F}^{p q}=\frac{1}{8 \pi \mu} \frac{\sinh \mathrm{D}_{p}}{\mathrm{D}_{p}} \frac{\sinh \mathrm{D}_{q}}{\mathrm{D}_{q}} \boldsymbol{J}\left(\boldsymbol{x}_{p}-\boldsymbol{x}_{q}\right)
$$

The operator $\mathrm{D}_{p}$ has been defined in $(2.4 b)$, which reduces to

$$
D_{p}^{2}=c_{p}^{2}\left[\left(d_{p} \cdot \nabla\right)^{2}+\left(1-e_{p}^{2}\right) / e_{p}^{2} \nabla^{2}\right]
$$

for axisymmetric ellipsoids. The non-local description (3.3a) and the infinite operator series (3.3 $b$ ) are fully equivalent (Kim 1986; Kim \& Arunachalam 1987). The former, however, is to be preferred for numerical evaluation since $(3.3 b)$ converges poorly, except when the particles $p$ and $q$ are very distant (Claeys 1991).

In the case of a force-free spheroid aligned in the principal direction of extension of an axisymmetric straining flow, Chwang \& Wu (1975) construct an exact solution to the Stokes equations using a parabolic distribution of dipoles along the symmetry axis of the particle, complemented by a biquadratic profile of octupoles reflecting its finite thickness. Slender-body theory, on the other hand, represents the rod as a line distribution of Stokeslets whose magnitude varies linearly with position (Batchelor $1970 \mathrm{~b}$ ). Both results may be reconciled by partial integration, as shown in Appendix A. Although the latter representation (A 2) seems 'messier' than Chwang \& Wu's result (A 1), it turns out to be useful when computing the interactions of fibres in close proximity to one another. By assigning the largest weight to the ends of the particle, this formulation emphasizes the elongated nature of the rods, in contrast to the solution (A 1), where the density of the dipoles vanishes at the extremities. In practice, therefore, the seemingly more complex slender-body representation is advantageous for computational aims. Analogous arguments motivate our preference for the nonlocal representation (3.3a) over (3.3b) for numerical purposes.

For unbounded systems, the grand mobility tensor becomes, in principle, of infinite dimension. Periodic structures, however, in which the configuration of $N$ chosen spheroids is replicated on a lattice, are amenable to a simpler treatment, provided all 'images' of a given particle are subject to the same force and torquet (i.e. $\forall\{z\}, F_{p+\{z\}}=F_{p}$ with $F_{p+\{z\}}$ the force exerted by the particle at $\boldsymbol{x}_{p}+\boldsymbol{x}_{\{z\}}$, and a similar condition on $T_{p}$.) In these circumstances, the dynamics of the entire dispersion may be described by a $11 N \times 11 N$ mobility tensor $\tilde{M}$.

To calculate $\tilde{M}_{U F}^{p q}$ for such a periodic system, one must apply the technique sketched in $\$ 2$ to a force-free spheroid $p$ embedded in a lattice of spheroids $q$, each exerting a force $F_{q}$ on the fluid, which would otherwise be quiescent. The velocity of the test particle $p$ is thus given by (2.5) as

$$
\begin{aligned}
U_{p}= & \frac{2 n_{\phi}}{15 \mu}\left[2 \boldsymbol{Q}_{q}: \delta-\delta: \boldsymbol{Q}_{q}+\frac{1}{3}\left(\boldsymbol{A}_{p}: \delta\right) F_{q}-\frac{1}{6} \boldsymbol{A}_{p} \cdot \boldsymbol{F}_{q}+\frac{1}{3}\left(\boldsymbol{A}_{q}: \delta\right) \boldsymbol{F}_{q}-\frac{1}{6} \boldsymbol{A}_{q} \cdot \boldsymbol{F}_{q}\right] \\
& -\frac{1}{8 \pi \mu} \sum_{\{z\}} \frac{\sinh \mathrm{D}_{p}}{\mathrm{D}_{p}} \int_{A_{q+(2)}} \boldsymbol{J} \cdot \boldsymbol{\sigma} \cdot \mathrm{d} \boldsymbol{A} \\
& -\frac{n_{\phi}}{8 \pi \mu} \int_{V-V_{\mathrm{q}}}\left\{\left(1+\frac{1}{6} \mathrm{D}_{p}^{2}+\frac{1}{6} \mathrm{D}_{q}^{2}\right) \boldsymbol{F}_{q} \cdot \boldsymbol{J}+\boldsymbol{S}_{q}: \boldsymbol{K}+\boldsymbol{Q}_{q} \odot^{3} \nabla \nabla \boldsymbol{J}\right\} \mathrm{d} \boldsymbol{V} .
\end{aligned}
$$

$\dagger$ If the problem is formulated as a resistance rather than a mobility problem, the imposed translational and angular velocities must be the same for all 'images' when measured relative to the ambient flow. 
We have assumed that no external torque acts on the particles and implicitly restrict the summation to those particles of the lattice whose centres lie in $V$. The integral of the stress density on the surfaces $A_{q+\{z\}}$ may then be expanded into irreducible moments as shown by Claeys \& Brady (1993a). Concentrating on the direct contribution of the force $F_{q}$ to $U_{p}$, we find

$$
\begin{aligned}
& \tilde{M}_{U F}^{p q}=\frac{n_{\phi}}{45 \mu}\left[2\left(A_{p}: \delta\right) \delta-A_{p}+2\left(A_{q}: \delta\right) \delta-A_{q}\right] \\
& +\frac{1}{8 \pi \mu} \sum_{\{z\}} \frac{\sinh \mathrm{D}_{p}}{\mathrm{D}_{p}} \frac{\sinh \mathrm{D}_{q}}{\mathrm{D}_{q}} J\left(x_{p}-x_{q}-\mathrm{x}_{\{z\}}\right)-\frac{n_{\phi}}{8 \pi \mu} \int_{V-V_{\epsilon}}\left(1+\frac{1}{6} \mathrm{D}_{p}^{2}+\frac{1}{6} \mathrm{D}_{q}^{2}\right) J \mathrm{~d} V .
\end{aligned}
$$

The summation describes the mobility interactions of particle $p$ with all spheroids $q$ (see $(3.3 b)$ ). The other terms in (3.5) represent the backflow (see $\$ 2$ ). As shown by Beenakker (1986), the lattice sum is most efficiently evaluated by noting that

and writing

$$
J(x)=\left(\nabla^{2} \delta-\nabla \nabla\right)|x|
$$

$$
\frac{1}{8 \pi \mu} \frac{\sinh \mathrm{D}_{p}}{\mathrm{D}_{p}} \frac{\sinh \mathrm{D}_{q}}{\mathrm{D}_{q}} J\left(x_{p}-x_{q}-\mathbf{x}_{\{z)}\right)=M_{U F}^{p[q+\{z)]}=M_{1}^{p[q+\{z\}]}+M_{2}^{p[q+\{z)]}
$$

with

$$
M_{1}^{p q}=\frac{1}{8 \pi \mu} \frac{\sinh \mathrm{D}_{p}}{\mathrm{D}_{p}} \frac{\sinh \mathrm{D}_{q}}{\mathrm{D}_{q}}\left(\nabla^{2} \delta-\nabla \nabla\right)\left(r_{p q} \operatorname{erfc}\left(\delta r_{p q}\right)\right)
$$

and

$$
M_{2}^{p q}=\frac{1}{8 \pi \mu} \frac{\sinh \mathrm{D}_{p}}{\mathrm{D}_{p}} \frac{\sinh \mathrm{D}_{q}}{\mathrm{D}_{q}}\left(\nabla^{2} \delta-\nabla \nabla\right)\left(r_{p q} \operatorname{erf}\left(\mathscr{E} r_{p q}\right)\right) .
$$

We have introduced the succinct notation $r_{p q}=\left|x_{p}-x_{q}\right|$; the parameter $\mathscr{E}$ controls the relative magnitude of $\boldsymbol{M}_{1}^{p q}$ and $\boldsymbol{M}_{2}^{p q}$. The sum of $\boldsymbol{M}_{1}^{p[q+\{z\}]}$ over all $\{z\}$ is performed in real space, using a non-local formulation similar to $(3.3 a)$, while the lattice summation of $\boldsymbol{M}_{2}^{p[q+\{z]]}$ is best carried out in reciprocal space by applying the Poisson summation formula (Nijboer \& de Wette 1957). This ingenious technique, originally developed by Ewald (1921) in an effort to estimate the cohesive energy of ionic crystals, was first applied to the Oseen tensor (2.1) by Beenakker (1986). Brady et al. (1988) extended his approach and lifted the restriction $\left\langle\boldsymbol{F}_{q}\right\rangle=0$ that he had placed on the derivation. We refer to the original papers for details concerning this method. Brady et al. (1988) also demonstrated that the application of the Ewald summation technique to the grand resistance tensor correctly accounts for hydrodynamic interactions in spatially periodic dispersions of spheres, and yields expressions which correspond term by term with the 'renormalized' formulation obtained using O'Brien's ideas (such as (2.5)). The proof for suspensions of spheroids follows the same lines (Claeys 1991). Using the Ewald technique (3.7), equation (3.5) can be converted into

$$
\tilde{M}_{U F}^{p q}=\sum_{\{z\}} M_{1}^{p[q+\{z\}]}+\frac{1}{|B|} \sum_{\{\zeta\} \neq 0} \cos \left(k_{\{b\}} \cdot\left(x_{p}-x_{q}\right)\right) \hat{M}_{2}^{p q}\left(k_{\{\xi}\right),
$$

where $k_{15}=\zeta_{1} k_{1}+\zeta_{2} k_{2}+\zeta_{3} k_{3}$ are the points of the reciprocal lattice. The basis vectors $k_{i}$ are defined such that $k_{i} \cdot b_{j}=2 \pi \delta_{i j}$, with $\delta_{i j}$ the Kronecker delta. $\hat{M}_{2}^{p q}$ is the Fourier transform $\int \exp (-\mathrm{i} k \cdot r) M_{2}^{p q}(r) \mathrm{d} r$. As explained for spheres in Brady et al. (1988), one can show that the 'backflow' integral and the first term on the right-hand side of (3.5) (proportional to $n_{\phi}$ ) are cancelled by the limit as $\boldsymbol{k}_{(6)} \rightarrow 0$ of $\hat{M}_{2}^{p q}\left(\boldsymbol{k}_{(\xi)}\right)$ (Claeys 1991). The 
origin of the lattice in $\boldsymbol{k}$-space is thus omitted from the sum. In essence, (3.8) replaces the slowly converging lattice sum in (3.5) by two complementary summations: one consists of terms which decay rapidly with distance, namely as $\operatorname{erfc}\left(\mathscr{E} r_{p q}\right)$; the second is Fourier transformed and calculated in reciprocal space. In this way, exponential rates of convergence are achieved for summands which decay only algebraically with distance (Nijboer \& de Wette 1957). The parameter $\mathscr{E}$ in (3.7) must be chosen optimally to balance the convergence rate of both sums (Beenakker 1986; Claeys 1991).

For $p=q,(3.8)$ is replaced by

$$
\tilde{M}_{U F}^{p p}=K_{U F}^{p}+\sum_{\{z\} \neq 0} M_{1}^{p[p+\{z\}]}+\frac{1}{|B|} \sum_{\{\zeta\} \neq 0} \hat{M}_{2}^{p p}\left(k_{\{\zeta\}}\right)-\left.M_{2}^{p p}\right|_{r=0}
$$

in order not to include the self-term twice in the mobility interactions. All the elements of the grand mobility tensor (3.2) are constructed using similar lattice summations (Claeys 1991).

The grand mobility tensor for $N$ periodically replicated prolate spheroids is inverted to yield a 'fair-field' estimate of the resistance tensor $\mathscr{R}$. Lubrication interactions between close particles are then included in a pairwise additive manner, as in the version of Stokesian dynamics for a finite number of spheroids (Claeys \& Brady 1993a):

$$
\mathscr{R} \approx \boldsymbol{R}=\tilde{\boldsymbol{M}}^{-1}+\boldsymbol{R}_{\mathrm{lub}}
$$

The only caveat is that situations may now occur in which a rod is simultaneously in the vicinity of more than one image of another particle. This geometric complication, however, merely requires special care in the organization of the code, and does not add any conceptual difficulties. Also, unlike spheres, lubrication interactions between a spheroid and its own images (on a dense lattice) may result in a net torque or stresslet.

The validity and accuracy of the scheme used here to approximate the grand resistance tensor, viz. using pairwise additivity of velocities to form a far-field estimate of the mobility tensor, and pairwise additivity of forces to preserve the large, localized lubrication stresses between close pairs of particles is discussed at length elsewhere (Claeys \& Brady 1993a; Durlofsky et al. 1987). We shall not repeat these arguments here. Dynamic simulations simply solve (3.1) for the motion of the particles by balancing the hydrodynamic and external forces and torques, and integrate the trajectories to update the microstructure. Because the spheroids are non-Brownian in this work, their paths are fully deterministic. The intricacies associated with thermal motion can be addressed by the methods developed for spheres (Bossis \& Brady 1989), and form a subject for further study. Since $\tilde{\boldsymbol{M}}$ is determined by the configuration of the particles, it needs, in principle, to be evaluated at each time step. This may be deferred in some instances to maximize computer time rentability, as discussed in Durlofsky et al. (1987).

\section{Calculation of hydrodynamic transport properties via Stokesian dynamics}

This section recapitulates the way hydrodynamic transport properties are evaluated in Stokesian dynamics (Brady \& Bossis 1988; Phillips, Brady \& Bossis 1988 a, $b$ ). These properties include the sedimentation rate of a dispersion, the self-diffusivities and the stress in a suspension, the hindered diffusion coefficients in porous media and the permeability of a fixed bed of particles. At the outset, let us emphasize that only the short-time limit of these properties is calculated in this work, since we do not track 
the evolution of the microstructure under influence of the flow. This asymptote can be accessed experimentally by dynamic light scattering or by rapid oscillatory rheometric measurements (at a frequency too high to affect the particle configuration in the suspension); the permeability, of course, is not frequency dependent (provided the quasi-stationary Stokes equations remain valid at the frequencies of interest).

The stress in the suspension is very often the quantity of most interest to the fluid dynamicist. For non-Brownian spheroids not subject to interparticle forces, the hydrodynamic stress $\Sigma$ of a suspension undergoing a shearing motion characterized by the rate of strain $E_{x}$ is given by (Batchelor $1970 a$ )

$$
\Sigma=-p_{\mathrm{erf}} \delta+2 \mu \boldsymbol{E}_{\infty}-n_{\phi}\langle\boldsymbol{S}\rangle .
$$

The first term is an isotropic term, which is irrelevant in incompressible media. The second term represents the Newtonian fluid phase response. The final contribution to the hydrodynamic stress comes from the particles, and is proportional to the mean stresslet $\langle\boldsymbol{S}\rangle$, which can be calculated from (3.1) as

$$
\begin{aligned}
\langle\boldsymbol{S}\rangle & =\left\langle\boldsymbol{R}_{S \mathscr{U}} \cdot\left(\mathscr{U}-\mathscr{U}_{\infty}\right)-\boldsymbol{R}_{S E}: \boldsymbol{E}_{\infty}\right\rangle \\
& =\left\langle\boldsymbol{R}_{S \mathscr{U}} \cdot \boldsymbol{R}_{\mathscr{F} \mathscr{U}}^{-1} \cdot \boldsymbol{R}_{\mathscr{F E}}-\boldsymbol{R}_{S E}\right\rangle: \boldsymbol{E}_{\infty} .
\end{aligned}
$$

In going from $(4.2 a)$ to $(4.2 b)$, we have assumed that the particles are freely suspended. The vector $\mathscr{U}$ lumps the translational and angular velocities of all particles; hence $\boldsymbol{R}_{S \psi t}$ stands for the $5 N \times 6 N$ tensor in the bottom left corner of $\mathscr{R}$ (see (3.1)) and includes both $\boldsymbol{R}_{S U}$ and $\boldsymbol{R}_{S \boldsymbol{\Omega}}$. Similarly, $\mathscr{F}$ groups the forces and the torques. The averages in the previous equations are taken over all particles, as well as over several realizations of the microstructure if applicable. The hydrodynamic stress in systems which are macroscopically isotropic is characterized by one coefficient (the effective viscosity of the medium), and additional averaging over the elements of the stress tensor $\boldsymbol{S}$ is possible, and recommended to obtain better statistics (since the five elements of $\boldsymbol{S}$ can be considered independent measures of the viscosity). In an extensive study of the multipole moment expansion technique for Stokes flow in random hard-sphere dispersions, Ladd (1990) has shown that the viscosity is relatively insensitive to the number of particles used in the simulation, and that the moment expansion converges rapidly, with meaningful results when the series is truncated at the level of stresslets (as done here), and little change beyond the octupole. Indeed, Phillips et al. (1988 a) report excellent agreement using Stokesian dynamics with the experimentally measured highfrequency limit of the effective viscosity for monodisperse hard-sphere dispersions (van der Werff et al. 1989).

To obtain the sedimentation velocity $\boldsymbol{V}$ of a suspension, we consider a collection of spheroids subject to gravity. For clarity, we shall assume that the dispersion is monodisperse, in which case all forces are equal. This is a mobility problem, which is solved by inverting (3.1) to obtain

$$
\left\langle\left(\begin{array}{c}
\boldsymbol{V} \\
\boldsymbol{W}
\end{array}\right)\right\rangle=\left\langle\boldsymbol{R}_{\mathscr{F} U}^{-1} \cdot\left(\begin{array}{l}
\boldsymbol{F} \\
0
\end{array}\right)\right\rangle=\left\langle\left(\begin{array}{ll}
\boldsymbol{R}_{F U} & \boldsymbol{R}_{F \Omega} \\
\boldsymbol{R}_{T U} & \boldsymbol{R}_{T \Omega}
\end{array}\right)^{-1} \cdot\left(\begin{array}{l}
\boldsymbol{F} \\
0
\end{array}\right)\right\rangle,
$$

or, writing out the contribution of each particle,

$$
\left\langle\left(\begin{array}{c}
\boldsymbol{V}_{p} \\
\boldsymbol{W}_{p}
\end{array}\right)\right\rangle=\left\langle\sum_{q-1}^{N}\left(\boldsymbol{R}_{\mathscr{F} U}^{-1}\right)^{p q} \cdot\left(\begin{array}{c}
\boldsymbol{F}_{q} \\
0
\end{array}\right)\right\rangle
$$

The averaging occurs over all particles $p$ in the unit cell, as well as over several realizations of the microstructure if desired. In most cases (including isotropic 
samples), $W$ is zero because the sedimentation process does not induce non-skewed particles to rotate in a systematic sense. Special microstructures can be conceived, however, which yield a non-zero average angular motion of the spheroids under the action of gravity. The collective diffusion coefficient, measuring the particle flux in response to a concentration gradient, is closely related to the sedimentation rate, and can be directly derived from it (Batchelor 1976). Unfortunately, these properties are known to display a strong system-size dependence, with the influence of periodicity decreasing very slowly (as $N^{-\frac{1}{3}}$ ) (Phillips et al. 1988a). Ladd (1990) devised a procedure to correct for this effect, which is very effective for dispersions of spheres. He also demonstrated, however, that many force moments need to be retained in the description of the particle dynamics in order to achieve quantitative accuracy for the sedimentation rate, even if lubrication interactions are explicitly included. Recent work by Revay \& Higdon (1992), however, indicates that truncation at the dipole level can give accurate sedimentation velocities if sufficient statistics are obtained in order to properly extrapolate to infinite $N$.

The self-diffusion tensor of a particle can be evaluated from the Stokes-Einstein formula as

$$
\langle\boldsymbol{D}\rangle=k T\left\langle\left(\boldsymbol{R}_{\boldsymbol{F} q}^{-1}\right)^{p p}\right\rangle,
$$

which in essence shows a direct proportionality between $D$ and the velocity of a tracer subject to a force in a suspension of force-free particles (' $k$ ' is the Boltzmann constant and $T$ the absolute temperature). In random monodisperse systems, the averaging usually occurs over all particles and several realizations of the microstructure. As noted by Phillips et al. (1988a), the diffusivity also displays a strong system-size dependence. Indeed, due to the spatially periodic nature of the dispersion, all images of the tracer particle are also subject to a force; hence the system de facto considers a simple lattice of spheroids sedimenting through $N-1$ lattices of neutrally buoyant particles. The effect of the periodically replicated force is long-ranged and introduces the $N^{-\frac{1}{3}}$ scaling. For random dispersions of hard spheres, Ladd (1990) successfully factored out this undesirable dependence by heuristically adapting the low- $n_{\phi}$ correction, known analytically (Hasimoto 1959), for the increase in viscosity occurring at higher concentrations (see also Phillips et al. 1988b). He also pointed out that the moments expansion technique is very adequate to evaluate this property and converges rapidly, provided lubrication interactions are included, as done here.

Hindered diffusion coefficients describe the mobility of a test particle in a fixed bed of other particles. We again rely on the Stokes-Einstein formula, which now becomes:

$$
\left\langle\boldsymbol{H}_{p}\right\rangle=k T\left\langle\left(\boldsymbol{R}_{F U}^{p p}\right)^{-1}\right\rangle \text {. }
$$

The effect of the long-ranged periodicity is partly screened in porous media (cf. the analysis of the Brinkman equation by Stokesian dynamics (Durlofsky \& Brady 1987)), resulting in a weak, at most $O\left(N^{-1}\right)$-dependence for this property (Phillips et al. 1988b). Also, as for self-diffusion and viscosity, the hindered diffusivity is expected to converge rapidly with the number of stress moments if lubrication interactions are added explicitly.

The permeability $\boldsymbol{K}$ of a fibrous bed relates the macroscopic pressure gradient to the mean velocity of the fluid in Darcy's law:

$$
\nabla p=-\boldsymbol{K}^{-1} \cdot\langle\boldsymbol{u}\rangle \text {. }
$$

It can be evaluated by simulating a uniform flow past a collection of immobile spheroids. From an overall force balance over a representative volume of the 
dispersion, it is clear that $\nabla p=-n_{\phi}\langle F\rangle$ (Brady et al. 1988). Therefore the resistivity $K^{-1}$ of a porous medium is given by

$$
\boldsymbol{K}^{-1}=n_{\phi}\left\langle\sum_{q} \boldsymbol{R}_{F U}^{p q}\right\rangle
$$

Since lubrication effects are nil for permeability calculations, the contribution of higher multipoles, which becomes sizeable at high packing fractions, can only be captured in the framework of Stokesian dynamics by including them at the stage of the mobility tensor. Many qualitative features of the dependence of $\boldsymbol{K}$ on concentration and aspect ratio, however, can adequately be reproduced using only 11 unknowns per particle, as done in this work. Artifacts due to periodicity are absent provided the Brinkman screening length (which scales as $1 / n_{\phi}^{\frac{1}{2}}$ ) is small compared to the size of the unit cell (Phillips et al. 1988b). This condition is easily satisfied except at very low solid volume fractions.

\section{Comparison of spheroids with strings of beads}

One of the major advantages of the simulation technique outlined here is that it only uses 11 unknowns per particle, yet claims to reproduce hydrodynamic interactions between the rods quite well. In order to check how adequately Stokesian dynamics renders the effect of the spheroids on the flow, we compared the properties of a crystal of spheroids with those of a lattice of spheres rigidly connected into strings. Each array contained 19 aligned, equidistant spheres. Since each bead of the arrangement has 11 degrees of freedom, this representation of an 'elongated' body captures more stress moments than only the total force, torque and stresslet exerted by the composite object. A crystal of strings was constructed by centring the chains on the nodes of a hexagonal grid with the normal along the direction of alignment of the 'rods', and stacking these sheets of particles directly on top of one another such that the gap between the layers equals the smallest separation between the arrays within each layer. The properties of this lattice were calculated using a version of Stokesian dynamics dedicated to spherical particles (Brady et al. 1988), and verified with our program in the limit of zero eccentricity. The single-string contribution $\eta_{T}$ to the extensional viscosity (i.e. the stresslet exerted by the rod in a uniaxial straining flow about the direction of alignment of the particles) is given by the solid line in figure 1 . We then matched this component of the stresslet $\left(\boldsymbol{S}_{p}: \boldsymbol{d}_{p} \boldsymbol{d}_{p}\right)$ for the string of beads at infinite dilution to that exerted by one of two isolated spheroids, the first having the same length as the composite rod (20 sphere diameters), but a different aspect ratio $\left(r_{p}=11.4\right)$, the other having the same aspect ratio $\left(r_{p}=20\right)$, but a longer axis $(a=21.93 R$, with $R$ the radius of the spheres). Both shapes are displayed in the insert of figure 1 . The properties of crystalline fibre dispersions, constructed by replacing each string by one of these spheroids, were then computed using Stokesian dynamics, as described in $\$ 3$, but suppressing lubrication effects in order to isolate the far-field interactions, which are of most interest here. Since both ellipsoids are considerably more voluminous than the string of beads (even the slender spheroid takes up about $40 \%$ more room), it is important to compare $\eta_{T}$ at the same number density of rods, rather than at equal packing fractions. Figure 1 shows that the data for the lines of spheres lie in between those for the lattices of spheroids. At each concentration, there therefore exists an ellipsoid whose geometry approximates that of the string of beads, and which has the same extensional viscosity at infinite dilution and at the chosen number density. More importantly, the version of 


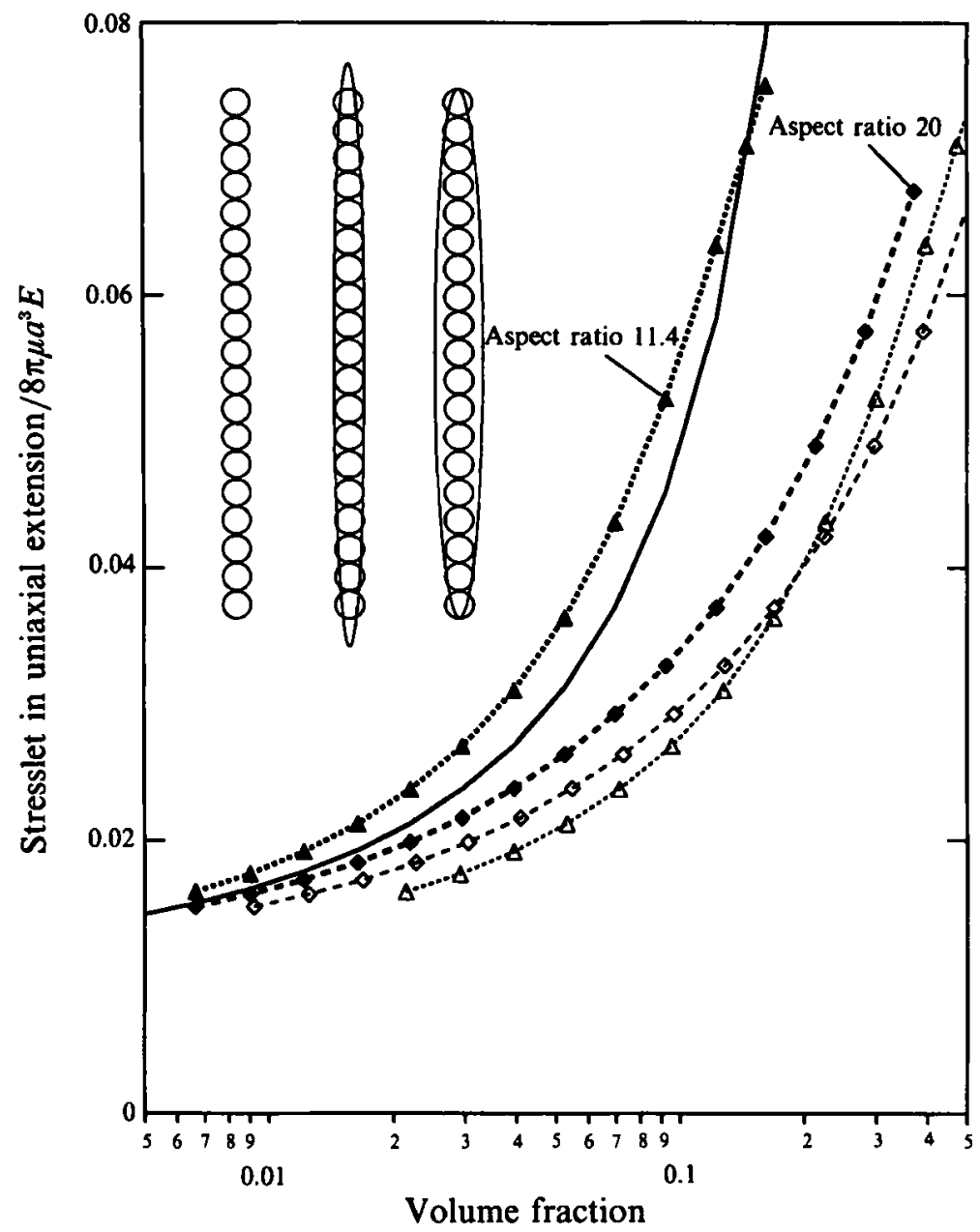

FIGURE 1. Comparison of the stresslet $\eta_{T}$ for crystals of spheroids with the result for a lattice of beads rigidly connected into strings. $E$ is the extensional rate of the uniaxial straining flow about the direction of alignment, to which the dispersion is subjected. $a$ is the length of the largest semi-axis of the spheroids, and is chosen as $\mathbf{2 0}$ sphere radii for the string of beads. The solid line gives the stresslet for the arrays of spheres. The bold dashed lines (with the filled symbols) are plotted $v s$. the volume fraction of equivalent strings of spheres; in this case, the abscissa measures the number density of particles. The dashed lines with the open markers show $\eta_{T} v s$. the actual volumetric concentration of spheroids.

Stokesian dynamics which truncates the moments expansion of the stress density after the first two terms (keeping only the effect of the force, torque and stresslet) seems at the very least to capture the qualitative features of the concentration dependence of the transport properties for crystals of rod-like objects. This represents a considerable computational advantage, since only 11 unknowns describe the spheroids in this example, as opposed to 209 for the string of 19 spheres. As the time required to carry out the matrix inversion (which yields $\tilde{M}^{-1} \approx R$; see (3.10)) grows with the cube of the number of unknowns, this is very significant. Figure 1 also shows that the results for the spheroids agree quite well with each other when plotted vs. the actual volume fraction, and that the calculations were carried out to nearly close packing, even though the corresponding density of spheres was only moderate $(\sim 15 \%)$. In other words, at the point where the upper curve crosses the data for the string of beads in 
figure 1, the spheroids are nearly touching and lubrication stresses, if they were accounted for, would undoubtedly increase the extensional viscosity above that of the line of spheres. The agreement thus remains very good up to the highest possible density.

\section{Hydrodynamic transport properties of equilibrium hard-spheroid dispersions}

A fibre influences the hydrodynamics of the suspension in a fluid volume of $O\left(a^{3}\right)$ surrounding it, with $a$ the particle half-length (Batchelor 1971; Shaqfeh \& Fredrickson 1990). Thus, $n_{\phi} a^{3}$ is the relevant measure of concentration for all hydrodynamic transport properties (as well as for the thermal conductivity (Shaqfeh 1988) and the dielectric constant, by mathematical analogy (Bonnecaze 1991)). The density, on the other hand, scales with $n_{\phi} a^{3} / r_{p}^{2}$. For spheroids of aspect ratio 10 , these scales differ by two orders of magnitude. It is therefore conceivable that a sample with an extremely low volume fraction is relatively concentrated as far as viscous interactions are concerned, with characteristics differing radically from those of the fluid alone. Furthermore, the excluded volume of rods varies as $a^{3} / r_{p}$, a scaling intermediate between that of hydrodynamic effects and that of the volume fraction. At least three concentration regimes can thus be distinguished in dispersions of hard rods (Doi \& Edwards 1986): dilute systems $\left(n_{\phi} a^{3} \ll 1\right)$ in which the spheroids are essentially isolated; semi-dilute samples $\left(1<n_{\phi} a^{3}<r_{p}\right)$, where hydrodynamic interactions are dominant; and concentrated rod dispersions $\left(n_{\phi} a^{3}>r_{p}\right)$ with noticeable excluded volume effects. The transition from dilute to semi-dilute can be thought of as the onset of entanglements, i.e. the separation between the fibres becomes smaller than their largest dimension. This leads to hydrodynamic screening in semi-concentrated rod suspensions (Shaqfeh \& Fredrickson 1990; Claeys \& Brady 1993 b). The crossover to concentrated dispersions is even more dramatic in a sense, since the microstructure changes appearance, with the formation of liquid crystalline domains. This phase transition is accompanied by discontinuities in the macroscopic properties of the suspension. In particular, the system is no longer isotropic. (The hallmark of liquid crystalline phases is long-ranged molecular orientational order. This confers a directional dependence to all tensorial observables, such as diffusivities, stress/rate-ofstrain relations, permeability, conductivity, refractive index, etc.)

In this section, we report on numerical simulations tracking the properties of equilibrium suspensions of hard spheroids as a function of the solid content. The effect of hydrodynamic interactions is always visible as a deviation from the single-particle behaviour: any nonlinearity in the concentration dependence betrays the importance of viscous interactions. In some instances, we shall also detect the isotropic-to-nematic transition. ('Nematic' is the liquid crystalline phase favoured thermodynamically by systems of hard spheroids at sufficiently high densities (Frenkel, Mulder \& McTague 1984).) Although external fields - either electromagnetic (for susceptible particles) or mechanical (an imposed continuous deformation for example - can induce orientational order, in effect lowering the isotropic-to-nematic boundary (Thirumalai 1986; See, Doi \& Larson 1990), we shall focus on equilibrium microstructures only. The effect of the flow on the configuration is not accounted for. As explained in $\S 4$, this amounts to calculating the short-time or high-frequency limit of the transport properties. The microstructures may be thought of as being representative of Brownian dispersions (at zero Péclet number), even though the spheroids in our simulations are not subject to thermal motion and Brownian stresses are not computed. 
Before discussing the results, we pause for a moment to consider the geometric problem of placing $N$ spheroids inside the unit cell. Orientational order spontaneously appears in the samples at concentrations such that $n_{\phi_{c}} a^{3} / r_{p} \approx 1$ (Doi \& Edwards 1986). The scaling with inverse aspect ratio obviously follows from the fact that excluded volume is the driving force for the thermodynamic transition to liquid crystalline states. Nematics are thus formed at lower volume fractions for slender fibres than for short rods, since the critical concentration $\phi_{\mathrm{c}}=n_{\phi_{\mathrm{c}}} 4 \pi a^{3} / 3 r_{p}^{2}=O\left(4 \pi / 3 r_{p}\right)$, a decreasing function of aspect ratio. On the other hand, it is advisable to work with unit cells which are large enough to accommodate the spheroids in any orientation they may choose, so as not to bias the resulting microstructure by the shape and size of the periodic building block. It follows that its volume $V_{\text {cell }}>8 a^{3}$. Hence the number of particles needed to simulate dispersions in the transition region from isotropic to liquid crystalline is $N=O\left(n_{\phi_{\mathrm{c}}} V_{\text {cell }}\right)=O\left(8 r_{p}\right)$. The inversion of the grand mobility matrix to get a far-field approximate to the resistance tensor (as required by the algorithm of Stokesian dynamics $(\S 3)$ ) is an $O\left(N^{3}\right)$ operation, and evaluating the mobility interactions is a costly $O\left(N^{2}\right)$ step. It is therefore computationally advantageous to examine spheroids of moderate aspect ratio if we are primarily interested in the behaviour near the phase boundary. Note that hydrodynamic interactions, on the other hand, change the character of the response at $n_{\phi}=O\left(a^{-3}\right)$, and can therefore be studied using $N=O(8)$, a very manageable number, and independent of aspect ratio. A second incentive to restrict the aspect ratios examined in the simulations is that the mobility tensor gets increasingly ill-conditioned as the particles become more slender, reflecting the fact that fibres experience less viscous resistance than blunt bodies, and hence dissipate less energy. The nearly singular nature of $M$ for extremely anisometric rods makes the computations more critically sensitive to numerical inaccuracies. In view of these arguments, we concentrated most of our efforts (and available share of CPU-time) on the study of dispersions of hard ellipsoids of aspect ratio 6. However, the method was found to work well even at the highest aspect ratio considered $\left(r_{p}=100\right)$.

We pointed out in the previous paragraphs that the excluded volume of a rod exceeds its actual volume by a factor proportional to the aspect ratio. This implies that it is much harder to generate an 'isotropic' configuration of rods than a random assemblage of spheres at the same volume fraction. The equilibrium hard-ellipsoid configurations discussed in this section were generated by a Monte-Carlo method (the energy function being infinite whenever two particles overlap, and indifferent to particle location and orientation otherwise). The spheroids were initially placed on an efc-lattice $\nmid$ at the desired concentration. The unit cell was made 'as cubic as possible' within the constraint of the imposed number of particles. In particular, a box shape standardization algorithm (Claeys 1991) guarantees that all angles inscribed between the edges of the unit cell lie between $\frac{1}{3} \pi$ and $\frac{2}{3} \pi$, thus preventing the accidental use of very skewed modules which would complicate the geometric analyses, and might bias the particle configuration. This places absolutely no restriction on the type of microstructures that can be examined. The particles are then subject to random translational and rotational displacements in the manner proposed by Frenkel \& Mulder (1985), rejecting all moves which cause spheroids to overlap. The 'quick'

$\dagger$ This structure is a face-centred crystal of parallel spheroids, dilated from closest packing such that the superficial separation between nearest neighbours is the same in three non-planar directions. The particles are oriented so that the direction of alignment is a 6-fold axis of symmetry for the lattice (Claeys \& Brady 1993b). 
Perram-Wertheim criterion (Perram \& Wertheim 1985; Allen, Frenkel \& Talbot 1989) was used to screen most of the potentially offending pairs, followed by the more elaborate test for overlap due to Vieillard-Baron (1970) if the first check was inconclusive. The Monte-Carlo procedure can be proven to sample configuration space evenly (Metropolis et al. 1953), producing representative snapshots of the equilibrium microstructures of hard-particle dispersions. We double-checked our method by verifying that the centres of mass of the spheroids wander diffusively as a function of the number of random displacements. We also found that hard-sphere suspensions generated in the same manner possess radial distribution functions in excellent agreement with the Percus-Yevick equation (Smith \& Henderson 1970; Perry \& Throop 1972; Verlet \& Weis 1972), except of course for the effects of periodicity. The values of the transport coefficients given below are averages over at least 6 to 8 different realizations of each dispersion of spheroids (at every concentration and for each aspect ratio). Only monodisperse samples were considered. Readers wishing to obtain information about particular aspect ratios or concentrations not discussed in this article should feel free to contact the authors. Additional data on equilibrium dispersions are tabulated in Claeys' thesis (1991) for $r_{p}=6$ and $r_{p}=20$, and results for other aspect ratios are available. Alternatively, a version of the program (in FORTRAN) may be requested.

Stokesian dynamics is a very efficient numerical technique, introducing only 11 unknowns per spheroid. Furthermore, all the hydrodynamic transport properties are calculated simultaneously, since the same information is exploited to solve for the sedimentation rate, the diffusivities and the rheology of the suspension (\$4). Computer time requirements are moderate, increasing with concentration and aspect ratio (i.e. with the number density of particles) at a given $N$. For a dispersion of spheroids with aspect ratio 6 at a volume fraction of $10 \%$ with 50 particles per unit cell, about $25 \mathrm{CPU}$-minutes are needed for each configuration on a SUN4 workstation.

\subsection{Diffusivities}

The diffusion tensor of an isolated axisymmetric rod is fully determined by four coefficients, which characterize its translational and rotary mobilities for motions parallel and perpendicular to the centreline. In an arbitrary dispersion, however, each fibre's environment is locally anisotropic and this simple structure for the diffusivity tensor is lost for each particle individually. Alternatively, on a global scale, the microstructure of an isotropic suspension is homogeneous and the rods do not diffuse in any preferred direction on average; neither is there any systematic correlation between centre-of-mass motions and reorientations. From a macroscopic perspective, the translational and rotational transport processes can thus be described by one scalar each. Yet we can define an average self-diffusivity parallel to the rod axis, $D_{\|}^{\text {tr }}$, as the mean projection of the diffusion tensor of each spheroid onto its director. With the Stokes-Einstein relation (see (4.5)), we thus set:

$$
\left\langle D_{\|}^{\mathrm{tr}}\right\rangle=k T\left\langle\left(\boldsymbol{R}_{\xi \Psi}^{-1}\right)_{U F}^{p p}: \boldsymbol{d}_{p} \boldsymbol{d}_{p}\right\rangle,
$$

and, for motions perpendicular to the fibre,

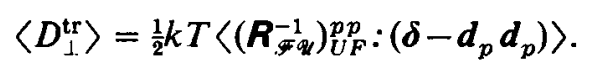

The rotational analogues obviously use the $\Omega L$ (bottom-right) portion of $\left(\boldsymbol{R}_{\mathscr{F} \text { ty }}^{-1}\right)^{p p}$. For 


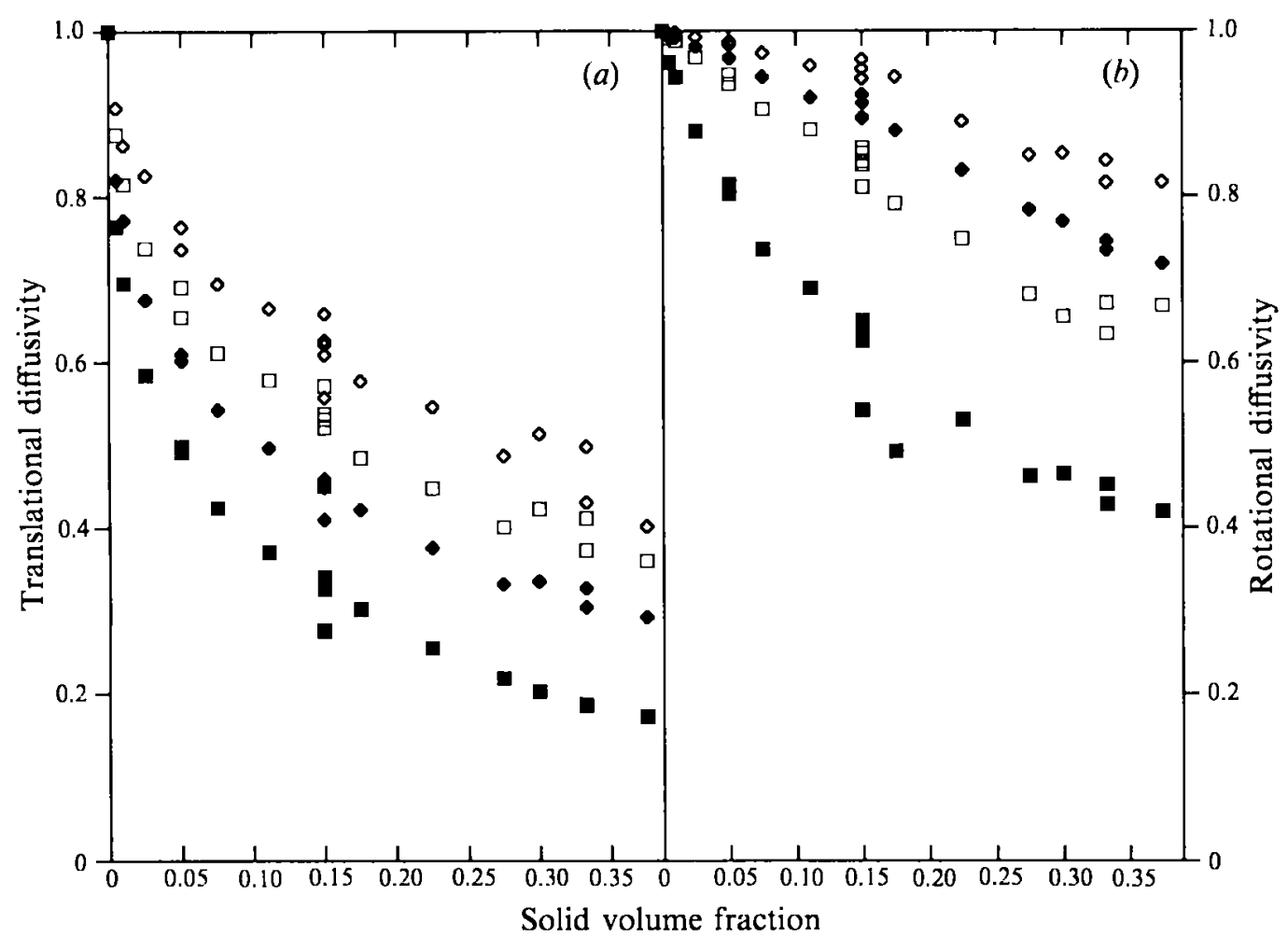

FIGURE 2. (a) Translational and $(b)$ rotational diffusivities of equilibrium dispersions of prolate spheroids of aspect ratio 6 . The open symbols give the short-time self-diffusivity: $\diamond D_{1} ; \square D_{\perp}$. The filled symbols are for the corresponding hindered diffusion coefficients: $H_{\|} ; \square H_{\perp}$.

an infinitely dilute monodisperse random dispersion, these definitions agree with the single-particle characteristics.

Figure 2 shows, for equilibrium dispersions of spheroids of aspect ratio 6, that motions perpendicular to the rod axis are affected more significantly by hydrodynamic interactions at a given concentration than diffusivities parallel to the centreline. This agrees with intuition, since they displace more fluid, creating a stronger flow field which is opposed by the surrounding particles. In essence, this expresses the notion that the 'hardest' motions in an unbounded fluid (characterized by the smallest value of the diffusivity) are also most sensitive to increases in the viscous resistance of the medium due to the presence of obstacles. Upon freezing of the microstructure to calculate hindered diffusion coefficients, this effect is accentuated and the sharpest drops are noted for $H_{\perp}^{\mathrm{tr}}$ and $H_{\perp}^{\mathrm{ro}}$. This may seem to agree with the basic assumption of reptation theory (de Gennes 1971; Doi \& Edwards 1986), but it must be emphasized that only the short-time limits of the transport properties are calculated here. The reduction in the mobility is due solely to hydrodynamic interactions, not to the 'caging' effect on which reptation theory is founded. Steric hindrance only enters the simulation through lubrication stresses, for the given instantaneous configuration of the particles. In addition, it is apparent that the assumption that $H_{\|}^{\text {tr }}$ remains equal to its free solution value (independent of concentration) is unwarranted, since it has already decreased by half in a dispersion containing $10 \%$ by volume of solid material.

The system of $\sim 60$ periodically replicated prolate spheroids of aspect ratio 6 considered here undergoes a phase transition to a nematic crystal (with a long-ranged 


$\begin{array}{ccccc}N & D_{\perp}^{\text {er }} & D_{\|}^{\text {er }} & D_{\perp}^{\text {ro }} & D_{\|}^{\text {ro }} \\ 25 & 1.35 & 2.27 & 4.82 & 49.3 \\ 30 & 1.56 & 2.48 & 5.06 & 49.9 \\ 50 & 1.59 & 2.53 & 5.00 & 48.7 \\ 60 & 1.61 & 2.55 & 4.98 & 48.7 \\ 64 & 1.71 & 2.68 & 5.10 & 49.9 \\ N & H_{\perp}^{\text {er }} & H_{\|}^{\text {er }} & H_{\perp}^{\text {ro }} & H_{1}^{\text {ro }} \\ 25 & 0.822 & 1.67 & 3.22 & 47.2 \\ 30 & 1.02 & 1.87 & 3.86 & 47.7 \\ 50 & 0.977 & 1.84 & 3.75 & 46.3 \\ 60 & 0.982 & 1.83 & 3.72 & 46.2\end{array}$

TABLE 1. Effect of the number of particles $N$ per unit cell on the calculated diffusivities of equilibrium dispersions of spheroids of aspect ratio 6 at $\phi=0.15$. (See also figure 2.) The transport coefficients are made dimensionless by $k T /\left(8 \pi \mu a^{n}\right)$, with $n=1$ for the translational components and $n=3$ for the rotary motions. For comparison, the corresponding diffusivities at infinite dilution are $D_{\perp}^{\text {tr }}=2.991$, $D_{1}^{\text {tr }}=4.069, D_{1}^{\text {ro }}=5.939, D_{\AA}^{\text {ro }}=51.67$; in this limit, the hindered mobilities are equal to the selfdiffusion coefficients.

orientational order) at a concentration of about $30 \% . \dagger$ This (imperfect) alignment of the rods does not greatly influence the mobility of the particles, however, and only the translational self-diffusivities seem to increase slightly at the phase boundary. The upward shift is indicative of the tube dilatation associated with the ordering of the fibres (Doi \& Edwards 1986). Although not nearly as spectacular, it is reminiscent of the jump and maximum observed for the density variation of $D_{\|}^{\mathrm{tr}}$ in molecular dynamics simulations of fluids of hard ellipsoids past the isotropic-to-nematic transition (Allen 1990). That work, of course, neglected all viscous interactions and assumed free-flight dynamics between collisions. The influence of the order parameter on the motional freedom of the particles may, however, be analogous in both systems.

For very dilute isotropic dispersions of identical spheroids, one may write unequivocally that the laboratory-frame diffusion tensor

$$
\langle\boldsymbol{D}\rangle=D_{\|}^{\mathrm{tr}}\langle\boldsymbol{d} \boldsymbol{d}\rangle+D_{\perp}^{\mathrm{tr}}\langle\boldsymbol{\delta}-\boldsymbol{d d}\rangle=\left(\frac{1}{3} D_{\|}^{\mathrm{tr}}+\frac{2}{3} D_{\perp}^{\mathrm{tr}}\right) \delta .
$$

Although $\langle\boldsymbol{D}\rangle \neq\left\langle D_{n}^{\text {tr }}\right\rangle\langle d \boldsymbol{d}\rangle+\left\langle D_{1}^{\text {tr }}\right\rangle\langle\delta-d d\rangle$ in general, we have verified numerically that this relation holds well (to within $\sim 1.5 \%$ ) for isotropic suspensions up to the transition to the nematic state. Thus the variation of $D \approx\left(\frac{1}{3} D_{\|}^{\mathrm{tr}}+\frac{2}{3} D_{\perp}^{\mathrm{tr}}\right) \delta$ with volume fraction can easily be deduced from figure 2 .

The translational self-diffusivity of the spheroids in equilibrium dispersions increases with the number of particles per unit cell because the backflow generated by the periodically replicated unit force diminishes. The effect of $N$ on the rotary diffusion is less pronounced, being $O\left(N^{-1}\right)$ at most (Phillips et al. 1988a), and is masked by the statistical uncertainty (table 1). The dependence on $N$ is also weaker in fibrous beds, because the fixed network tends to screen the disturbance velocities, as explained in $\S 4$.

Recall from $\S 4$ that the influence of the number of particles $N$ per unit cell decays quite slowly, as $N^{-\frac{1}{3}}$, for the self-diffusivities. The data displayed in figure 2 have not

$\dagger$ The volume fraction at the transition lies slightly below the phase boundary found by MonteCarlo methods for fluids of hard ellipsoids (Frenkel \& Mulder 1985; Allen 1990 report $\phi_{\mathrm{c}} \approx 0.36$ for $r_{p}=5$ ). This lowering of the critical concentration is presumably a weak periodicity effect, enhanced by the relatively small size of the periodic box. For comparison, several hundred particles were used in the Monte-Carlo and molecular dynamics studies. 


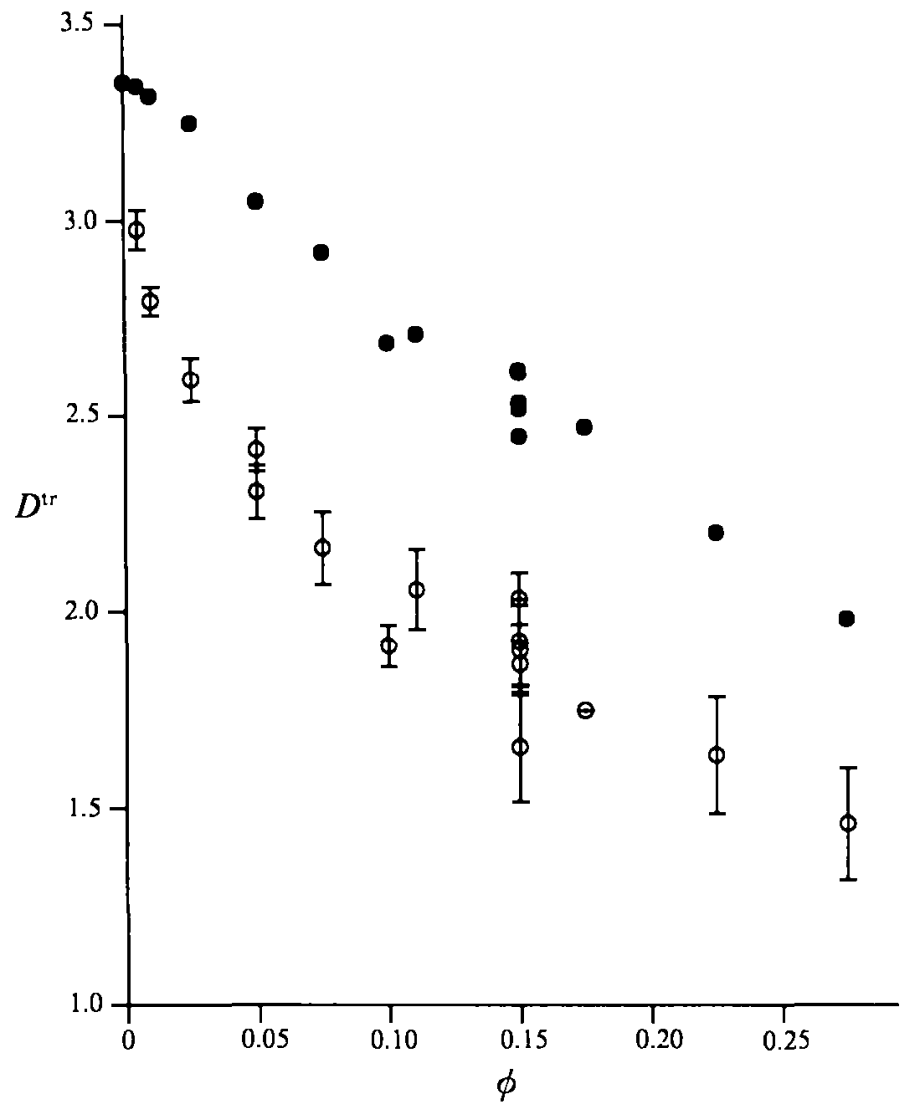

FiguRE 3. Corrected values of the translational self-mobility of spheroids of aspect ratio 6 in equilibrium dispersions. The diffusion coefficient is normalized by $k T / 8 \pi a \mu$. The open circles show the simulation data. The results, extrapolated to the thermodynamic limit by means of (6.2), are given by the solid symbols.

been corrected for this periodicity effect, and hence do not represent the thermodynamic limit. As stated in $\S 4$, the procedure proposed to extrapolate to $N \rightarrow \infty$ (Phillips et al. $1988 a$; Ladd 1990) subtracts the contribution of the periodically replicated images cosedimenting with the diffusive tracer. This effect is known asymptotically for dilute arrangements of spheres on a cubic lattice (Hasimoto 1959), but not for ellipsoids. Indeed, the numerical factor multiplying the $O\left(\phi^{\frac{1}{3}}\right)$ correction then not only depends on the shape of the unit cell (which is not cubic in general), but also on the orientation of the spheroidal axis relative to the basis vectors. We can, however, estimate the magnitude of the periodicity effect from our computations on efc-lattices (Claeys \& Brady $1993 b$ ) and adjust the data of figure 2 according to the expression

$$
D_{\left\{\phi_{0} ; N \rightarrow \infty\right\}}^{\mathrm{tr}}=D_{\left\{\phi_{0} ; N_{0}\right\}}^{\mathrm{tr}}+\left(D_{\{\phi>0\}}^{\mathrm{tr}}-V_{\left\{\phi=\phi_{0} / N_{0} ; \mathrm{efc}\right\}}^{\mathrm{tr}}\right) \frac{\mu\left(\phi_{0} / N_{0}\right)}{\mu\left(\phi_{0}\right)} .
$$

$\phi_{0}$ is the concentration of interest, and $N_{0}$ the number of particles in the simulation cell. The sedimentation rate $V^{\text {tr }}$ is understood to be that of an efc-crystal with density $\phi_{0} / N_{0}$, corresponding to the volume fraction of the images of the diffusing spheroid. As explained by Ladd (1990), the factor $\mu\left(\phi_{0} / N_{0}\right) / \mu\left(\phi_{0}\right)$ is introduced because the $N_{0}-1$ intervening particles attenuate the periodicity effect without altering its functional form (i.e. the images of the diffusive tracer sediment in a medium of higher effective viscosity 


$\begin{array}{cccc}\phi_{0} & N_{0} & D_{\mid N=N_{0}}^{\mathrm{tr}} & D_{\mid N \rightarrow \infty}^{\mathrm{tr}} \\ 0.05 & 30 & 2.31 & 3.049 \\ 0.05 & 50 & 2.42 & 3.047 \\ 0.15 & 25 & 1.66 & 2.45 \\ 0.15 & 30 & 1.87 & 2.61 \\ 0.15 & 50 & 1.90 & 2.53 \\ 0.15 & 60 & 1.92 & 2.52 \\ 0.15 & 64 & 2.03 & 2.62\end{array}$

TABLE 2. Corrected values of the translational self-diffusion coefficient of equilibrium dispersions of spheroids $\left(r_{p}=6\right)$. Formula (6.2) was used to extrapolate the simulation results to the thermodynamic limit $N \rightarrow \infty$ at a fixed concentration $\phi_{0}$.

than a dispersion with concentration $\phi_{0} / N_{0}$ ). This ratio of viscosities can be estimated from our results for random suspensions (see $\$ 6.3$ ). Figure 3 shows that the adjustment is substantial, especially at the lowest volume fractions (reflecting the dependence on $\left.\phi^{\frac{1}{3}}\right)$, and that the spread on the data obtained with different values of $N$ is reduced by the correction formula (6.2) (see also table 2). The steep decline of $D^{\operatorname{tr}}$ with $\phi$ noted for dilute dispersions in figure 2 thus merely reflects the periodicity of the microstructure introduced by the boundary conditions, and is absent in truly random systems. Indeed, the extrapolated values of $D^{\text {tr }}$ decrease approximately linearly with concentration, as observed experimentally for spheres (Ottewill \& Williams 1987; Pusey \& van Megen 1983; van Megen \& Underwood 1989; see also Phillips et al. 1988a). The variance reported for the self-diffusivities in figure 3 is representative for all the transport properties and reflects the actual spread of the individual mobilities as a result of the differing local environments experienced by each particle. The average values themselves are reproducible to within two significant figures (or within $5 \%$ in some cases), a much tighter bound than that suggested by the error bars.

Within the scatter of the data in figure $2(b)$, the rotational self-diffusivities, for which periodicity effects are much less pronounced, depend linearly on concentration. This behaviour was also observed in suspensions of hard spheres upon suppression of lubrication interactions (Phillips et al. 1988b). Note that lubrication effects are relatively weak in our simulations, since the dispersions are not at all close-packed. Moreover, recall that the minimum separation between the rods must be smaller than their shortest dimension, i.e. $o\left(a / r_{p}\right)$, in order for the asymptotic formulae of Claeys \& Brady (1989), which we use here for lack of better information, $\dagger$ to apply. Hence, in an arbitrary microstructure, only very few pairs of particles are ever close enough to experience lubrication forces.

Stretched exponential decays are often used to fit the $\phi$-dependence of the translational diffusion coefficients (White \& Dorion 1961; Wheeler et al. 1987; Phillies 1987). In particular, the law set forth by Cuckier (1983) on semi-heuristic grounds for the diffusivity of a sphere in a network of fibres, namely

$$
H_{\mid \phi}^{\mathrm{tr}}=H_{\mid \phi-0}^{\mathrm{tr}} \exp \left(-\kappa \phi^{\frac{1}{2}}\right)
$$

gives quite a satisfactory fit for the hindered translational mobility of a (periodically replicated) freely suspended particle in an otherwise frozen equilibrium distribution of spheroids (figure 4). Since fibrous beds can be described by a Brinkman equation (Spielman \& Goren 1968), the observed dependence on $\phi^{\frac{1}{2}}$ can be interpreted as a manifestation of hydrodynamic screening. Indeed, Cuckier's derivation of (6.3) relied

† The $O\left(\epsilon^{0}\right)$ correction to these formulae, with $\epsilon$ the non-dimensionalized gap width between the nearly touching surfaces, is unknown. The errors it introduces are not severe (Claeys 1991). 


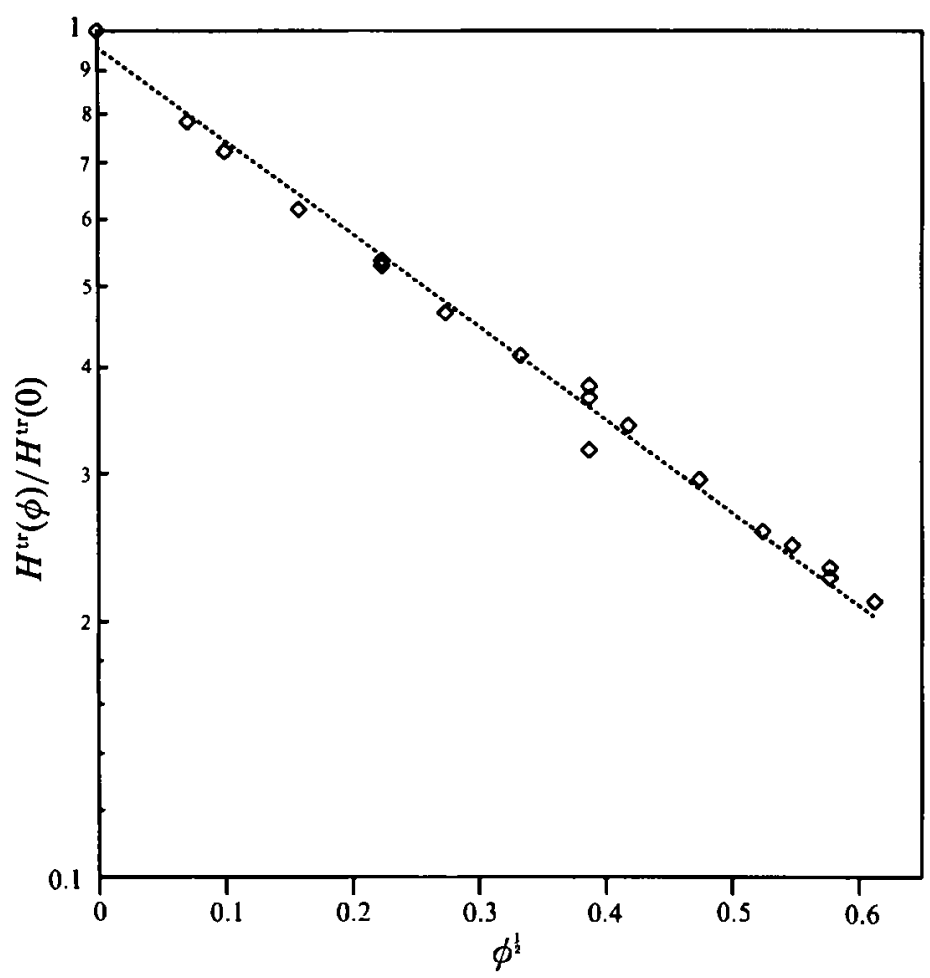

FIGURE 4. Semi-logarithmic plot of the hindered translational diffusivity in equilibrium dispersions of spheroids $\left(r_{p}=6\right) v s$. the square root of concentration. The diffusion coefficient is normalized by its value at infinite dilution.

on the concept of a screening length proportional to $\phi^{-\frac{1}{2}}$. The results of figure 4 thus confirm that our simulation technique adequately captures the many-body effects responsible for screening in fibrous media (see also Durlofsky \& Brady 1987). The influence of the particle shape on the degree of retardation (i.e. on $\kappa$ in (6.3)) is reported in figure 5. Concentration has been expressed as $r_{p}^{2} \phi=n_{\phi} \frac{4}{3} \pi a^{3}$. For rods of equal length, this may be thought of as the number density of spheroids. Alternatively, the abscissa can be interpreted as a count of the particles which interact hydrodynamically with the diffusing ellipsoid since, as mentioned earlier, the volume of the circumscribing sphere is a measure of the region in which the presence of a fibre affects the flow. We have chosen to illustrate the effect of the particles' eccentricity by means of the hindered translational diffusivity perpendicular to the rod axis, but the main characteristics of figure 5 are the same for all mobility coefficients. At a given number density, one notes from figure 5 that the most slender spheroids hinder each other's motion the least. In other words, hydrodynamic interactions between two rods of fixed length $a$ at a set centre-to-centre separation are weakest for the most slender particles. This should be clear intuitively since they occupy the least space and displace the smallest amount of fluid as they move. Note however that in terms of volume fractions, the trend is opposite, with the steepest drop in $H_{\perp}^{\text {tr }}$ (relative to the value at infinite dilution) being noted for the most elongated fibres. Indeed, at $n_{\phi} \frac{4}{3} \pi a^{3}=10$, the dispersion of spheroids of aspect ratio 6 contains $28 \%$ solid material, compared to $0.1 \%$ for the rod suspension with $r_{p}=100$. Also, the diffusivity of ellipsoids of aspect ratio 20 has dropped by half at $\phi=0.025$, but it takes twice this concentration to reach a similar decrease in a suspension of rods with $r_{p}=6$. 


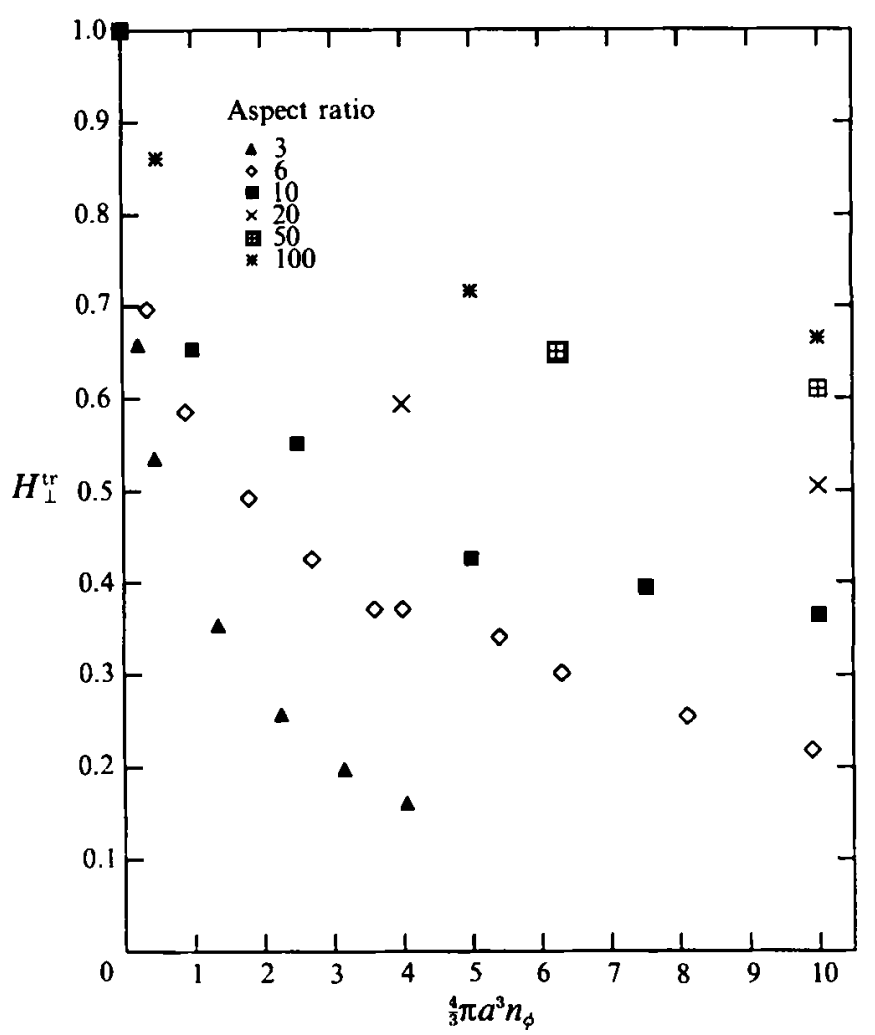

Figure 5. Hindered diffusion coefficient $H_{\perp}^{\mathrm{r}}$ vs. number density of particles for equilibrium dispersions of spheroids of various aspect ratios. The diffusivities are normalized by their values at infinite dilution. Each simulation typically used 60 particles per unit cell.

\subsection{Resistivity of fibrous media}

As explained in $\S 4$, Stokesian dynamics provides a way to calculate the permeability of fibrous packings at virtually no extra cost along with the diffusivities discussed in the previous subsection and the rheological properties of suspensions (examined below). Figure 6 depicts some simulation data on the resistivity of equilibrium dispersions of spheroids. The mean pressure gradient over the fixed bed of fibres increases with the packing density, as expected. Since a straightforward superposition of single-rod contributions would yield a linear variation with $\phi$, the curvature in figure 6 must result from hydrodynamic interactions. These cause the dependence on concentration to steepen. At a given volume fraction, the pressure drop across a fibrous bed increases with the aspect ratio of the spheroids (figure 6). This, of course, results from the higher number density of the slimmer ellipsoids.

The dashed lines in figure 6 show the equivalent pressure gradient, $n_{\phi} F$, calculated for spheroids of aspect ratio 6 from the mean component of the force along the rod's axis $\left(n_{\phi}\left\langle F_{\|}\right\rangle\right.$, lower curve) and perpendicular to it $\left(n_{\phi}\left\langle F_{1}\right\rangle\right.$, upper bound). The net resistivity of random dispersions lies between the two extremes, albeit somewhat closer to the highest limit, as expected. Also noted at concentrations above the isotropic-tonematic transition are the pressure drops for flow in the direction of alignment and at right angles to it. These values differ from the bounds because the ordering of the rods in the nematic phase is imperfect. It is easy to understand why structural deviations from ideality affect the permeability in the direction of the fibres the most, since any misalignment raises the pressure gradient in this case. The resistance to cross-flows, on 


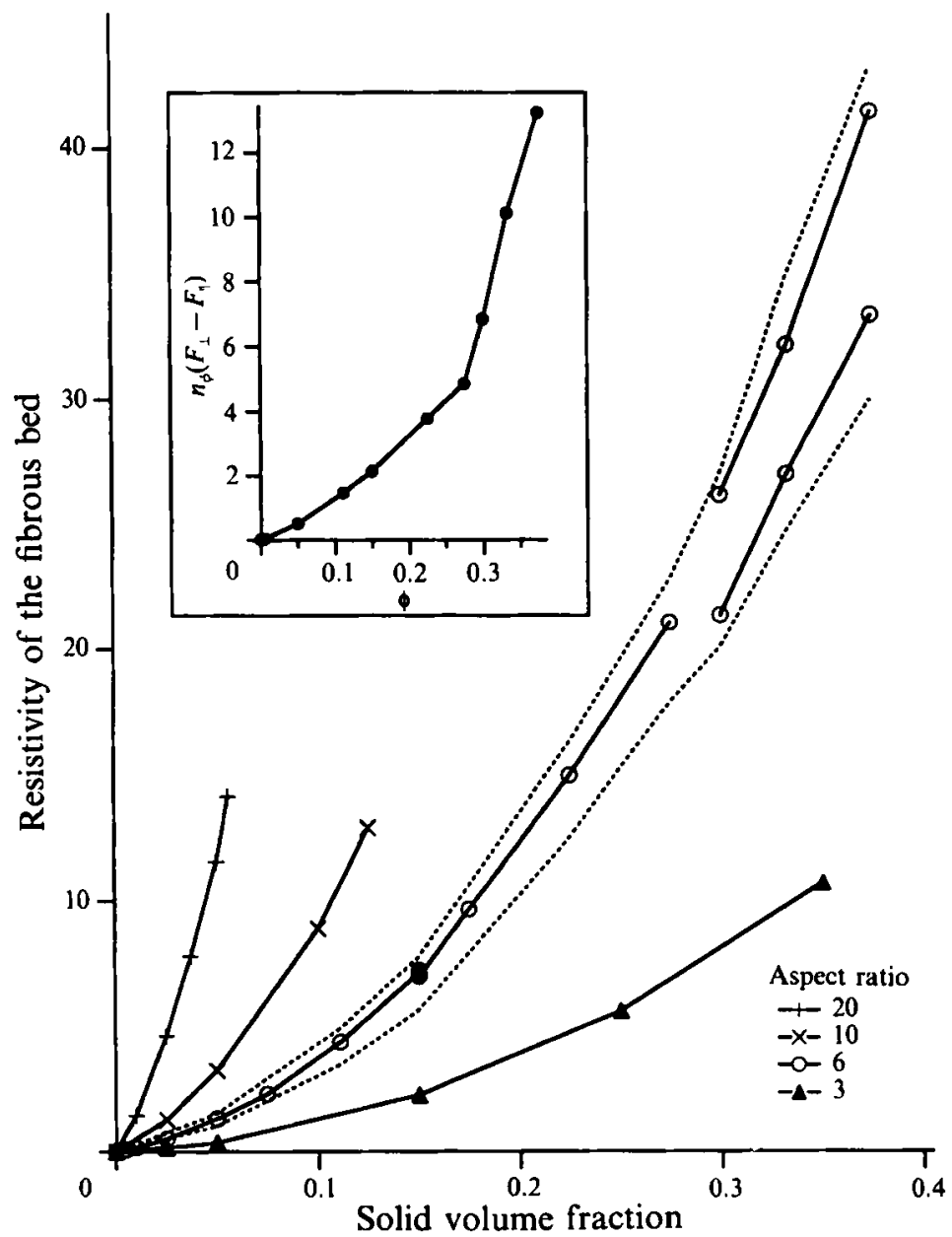

FIGURE 6. Resistivity of fibrous media, normalized by $6 \mu / a^{2}$. The insert, for $r_{p}=6$, clearly shows that the difference between the average component of the force supported by the rods along their axis and perpendicular to it is emphasized past the isotropic-to-nematic phase transition.

the other hand, is relatively indifferent to disorder in the plane perpendicular to the stream vector. The phase change at $\sim 30 \%$ also influences the bounds on the pressure gradient (the dashed curves of figure 6) inasmuch as the spread between them widens considerably more rapidly with increasing concentration past the transition. This behaviour follows from the definition of $\left\langle F_{\|}\right\rangle$and $\left\langle F_{\perp}\right\rangle$ as $\left\langle\Sigma_{q} R_{F V}^{p q}: d_{p} d_{p}\right\rangle$ and $\frac{1}{2}\left\langle\Sigma_{q} R_{F U}^{p q}:\left(\delta-d_{p} d_{p}\right)\right\rangle$ respectively (see also (4.8)). In isotropic dispersions, the fibres are arbitrarily oriented, and the influence of the rods $q$ on the force exerted by $p$ is statistically uncorrelated. In nematics, $d_{q}$ and $d_{p}$ are nearly aligned and all the spheroids of the dispersion act consistently on the resistance of particle $p$. Hence the difference between flows parallel and perpendicular to the fibre axis are accentuated in the liquid crystalline phase, and the bounds diverge.

\subsection{Viscosity and stress/rate-of-strain relations}

If the fibres are force-free rather than immobile, the rheology of the suspension is of interest. In disordered dispersions, for which all rod orientations are equally probable, the stress response to a rate of strain is Newtonian and can be characterized by one scalar, the effective viscosity. This quantity is plotted in figure 7 for equilibrium 


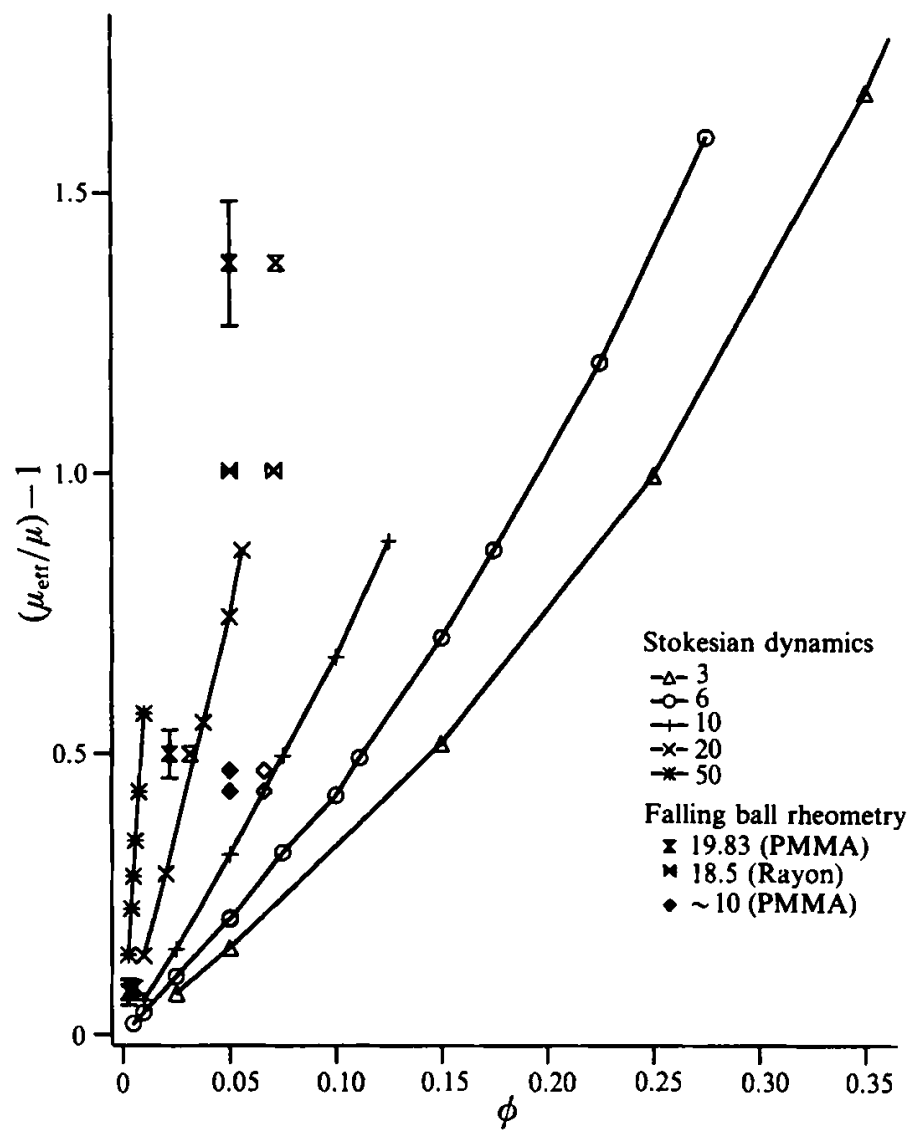

FIGURE 7. Viscosity of isotropic dispersions of spheroids. The experimental measurements reported are taken from Milliken et al. (1989) and Powell et al. (1989). The solid symbols are their raw data; the open symbols have been adjusted to fit an 'equivalent' spheroid.

microstructures of spheroids with aspect ratio ranging from 3 to 50 . The values reported are means of the particle stresslets over several realizations at each concentration. Typical simulations used 50 to 64 particles per unit cell. In addition, the six pertinent components of $\left\langle\boldsymbol{R}_{E E}\right\rangle=\left\langle\boldsymbol{R}_{S \Psi} \cdot\left\langle\boldsymbol{R}_{F \mathscr{G}}^{-1} \cdot \boldsymbol{R}_{F_{E}}-\boldsymbol{R}_{S E}\right\rangle\right.$ were also averaged as independent measures of the viscosity. The disproportionate enhancement of the viscosity by the addition of a few fibres to the medium (notice the steep slope of the concentration dependence for $r_{p}=50$ in figure 7) is explained by the fact that there are always some rods in an isotropic suspension which lie along the principal axes of extension of the flow. These components of the straining field are then very effectively counteracted by the rigid, unstretchable rods, generating the large stress response. The contribution of the fibres to the resistance of the suspension increases with the aspect ratio of the particles (figure 7), as would be expected from the dilute-limit expansion of the viscosity of fibrous dispersions. Indeed, at low concentrations, the Einstein correction formula for the stress in suspensions of randomly oriented spheroids becomes (Claeys 1991):

$$
\mu_{\mathrm{ett}}=\mu\left[1+\frac{6}{5} r_{p}^{2} \phi\left(\eta_{T}+2 \eta_{\|}+2 \eta_{\perp}\right)\right]
$$

with

$$
\eta_{T}=-\frac{2}{9} e^{3} \alpha_{5}, \quad \eta_{\|}=-\frac{2}{3} e^{3} \alpha_{4}, \quad \eta_{\perp}=-\frac{1}{3} e^{3}\left(\alpha_{1} \frac{e^{2}}{2-e^{2}}+\alpha^{*}\right) .
$$

The coefficients $\alpha_{1}, \alpha_{4}, \alpha_{5}$ and $\alpha^{*}$ are known functions of the eccentricity $e_{p}=\left(1-r_{p}^{-2}\right)^{\frac{1}{2}}$ 
of the generating ellipse (Kim 1985; Chwang \& Wu 1975), with the following asymptotes for slender spheroids $\left(e_{p} \rightarrow 1\right)$ :

$$
\begin{gathered}
\left.-\alpha^{5} \stackrel{e \rightarrow 1}{\longrightarrow \frac{1}{2}(2} \log \left(2 r_{p}\right)-3\right)^{-1}+O\left(r_{p}^{-2}\right), \\
-\left(\alpha_{1} \frac{e^{2}}{2-e^{2}}+\alpha^{*}\right) \stackrel{e \rightarrow 1}{\longrightarrow} r_{p}^{-2}+O\left(r_{p}^{-4}\right), \\
-\alpha_{4} \stackrel{e \rightarrow 1}{\longrightarrow \frac{1}{2} r_{p}^{-2}}+O\left(r_{p}^{-4}\right) .
\end{gathered}
$$

These limiting forms are accurate over a remarkably wide range of aspect ratios (Kim 1986; Claeys \& Brady 1993b). In essence, $\alpha_{5}$ gives the resistance of a rod to uniaxial extension about its symmetry axis; $\alpha_{4}$ corresponds to the apparent viscosity during simple shear (or hyperbolic straining) in a plane perpendicular to the fibre, and the combination $(6.5 b)$ represents the stress response to simple shearing flows with a velocity gradient parallel to the centreline of the ellipsoid.

The dependence of the effective viscosity on the slenderness of the particles can be attributed almost exclusively to the number density of spheroids, which grows as $r_{p}^{2}$ at equal volume fractions. The coefficients $\eta_{\|}$and $\eta_{\perp}$ decrease roughly as $r_{p}^{-2}$ (see $(6.4 c, d)$, thus cancelling the effect of the number density on the macroscopic shear stress, but $\eta_{T}$ is only weakly dependent on the particle shape (see $(6.4 b)$ ), and explains the rise in viscosity with increasing aspect ratio. Evidently, $\left(\mu_{\text {eft }} / \mu-1\right)$ does not quite vary as $r_{p}^{2}$ at a given $\phi$, unless $\eta_{T} \gg \eta_{\|}+\eta_{\perp}$, i.e. unless the fibres are very slender. The data in figure 7 support this assertion, since the relative increase in the effective viscosity is larger from $r_{p}=10$ to $r_{p}=20$ than from $r_{p}=3$ to $r_{p}=6$ at any given concentration, even though neither increase is even close to a quadrupling. This is also illustrated in figure 8(a), where the particles' contribution to the effective viscosity is plotted $v s$. the number density of particles. For rods of very high aspect ratio, the curves should almost collapse since their stresslet is then dominated by $\eta_{T}$, which depends only weakly on $r_{p}$ (namely as $\log ^{-1}\left(r_{p}\right)$ to a first approximation for dilute systems of very slender fibres). We see a much stronger dependence on the shape of the spheroids in figure 8(a), reflecting the non-negligible effect of $\eta_{\|}$and $\eta_{\perp}$ on the rheology of these dispersions. Although the curves in this figure seem perfectly straight on this doublelogarithmic graph, the linear plot of figure 7 clearly shows a slight upward bend, indicating modest deviations from dilute suspension behaviour.

Figure 9 more explicitly demonstrates that our simulations capture the nascent semidilute concentration regime at the highest volume fractions. Somewhat surprisingly, the dispersion containing $1 \%$ rods of aspect ratio 50 still responds hydrodynamically as if it were dilute, even though $n_{\phi} \frac{4}{3} \pi a^{3}=25$. Examining the rheological properties of more concentrated systems for this aspect ratio would require the use of a prohibitively large number of particles per unit cell. (The density of a suspension with 64 spheroids of $r_{p}=50$ in a periodic box of side $2 a$ is 0.0134 . Any increase of the volume fraction beyond this value at fixed $N$ violates the constraint that the smallest box dimension must be larger than the particle length.) Also drawn in figure 9 is the theoretical prediction of Shaqfeh \& Fredrickson (1990) for the hydrodynamic stress in semi-dilute suspensions of slender rods: $\dagger$

$$
\frac{\mu_{\text {eff }}}{\mu}-1=\frac{8 \pi a^{3} n_{\phi}}{45 \ln (1 / \phi)}\left(1-\frac{\ln \ln (1 / \phi)}{\ln (1 / \phi)}-\frac{0.2115}{\ln (1 / \phi)}\right)+O\left(\frac{n_{\phi} a^{3}}{\ln ^{3}(1 / \phi)}\right) .
$$

† Shaqfeh \& Fredrickson (1990) used $2 \pi a^{3} r_{p}^{-2} n_{\phi}$ instead of $\frac{4}{3} \pi a^{3} r_{p}^{-2} n_{\phi}$ for the volume fraction of rods. The numerical constant in (6.6) has been adjusted accordingly. 


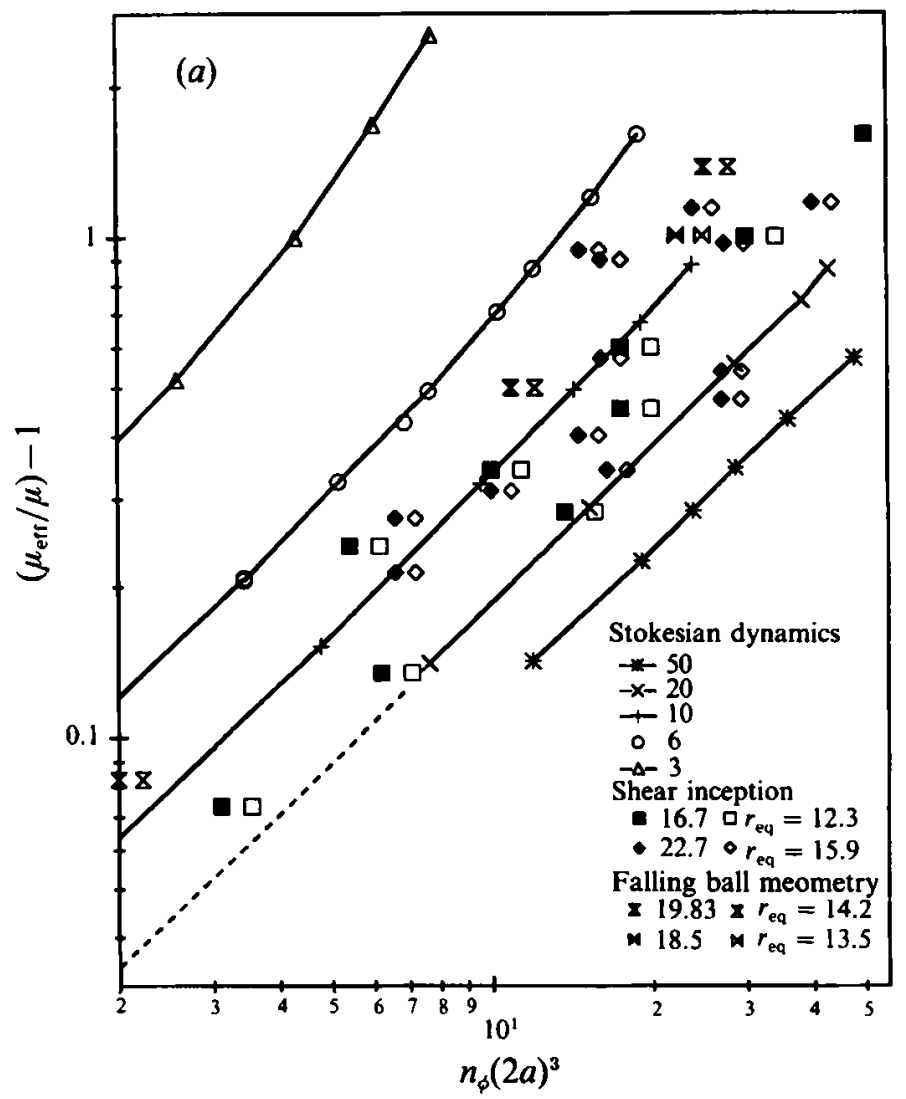

FIGURE 8(a). For caption see facing page.

Their analysis thus predicts that the stresslet varies as $\left[\log \left(\phi^{-1}\right)\right]^{-1}$ to a first approximation in the semi-concentrated range, whence the plot of $\eta_{T}^{-1} v s . \log (\phi)$ in figure 9. Our data are consistent with their result, but do not support it conclusively. We note, however, a definite transition from a dilute concentration range, where the stresslet is independent of the volume fraction, to a semi-dilute regime, in which the particle's resistance grows with $\phi$.

We mentioned earlier that equilibrium dispersions of hard spheroids with aspect ratio 6 undergo a phase transition to a liquid crystalline state above $30 \%$ by volume. Figure 10 demonstrates that the accompanying structural change causes an abrupt increase in the extensional viscosity (assuming that the principal direction of strain lies along the director of the nematic phase). This is easily explained by the fact that all particles are now oriented (on average) so as to oppose the attempted stretch most effectively. (The small graph appended to figure 10 shows $\left\langle P_{2}\left(d_{p} \cdot d_{q}\right)\right\rangle$ as a function of density, with $P_{2}(x)$ the second-order Legendre polynomial. The average runs over all pairs of rods in the unit cell. For an isotropic orientation distribution, $\left\langle P_{2}\right\rangle=0$, and $\left\langle P_{2}\right\rangle=1$ if all rods are perfectly aligned. The transition to a nematic phase is clearly visible.) The jump in $\mu_{\text {ext }}$ is not however nearly as large as might have been expected from a comparison with the extensional viscosity of efc-lattices (dashed line in figure 10 ; Claeys \& Brady 1993b). In order to test the hypothesis that this was due to the incomplete alignment of the rods, we also calculated the rheological properties of a few dispersions of parallel spheroids whose centres of mass were statistically uncorrelated, thus isolating the effect of orientational disorder. The results shown in figure 10 prove 


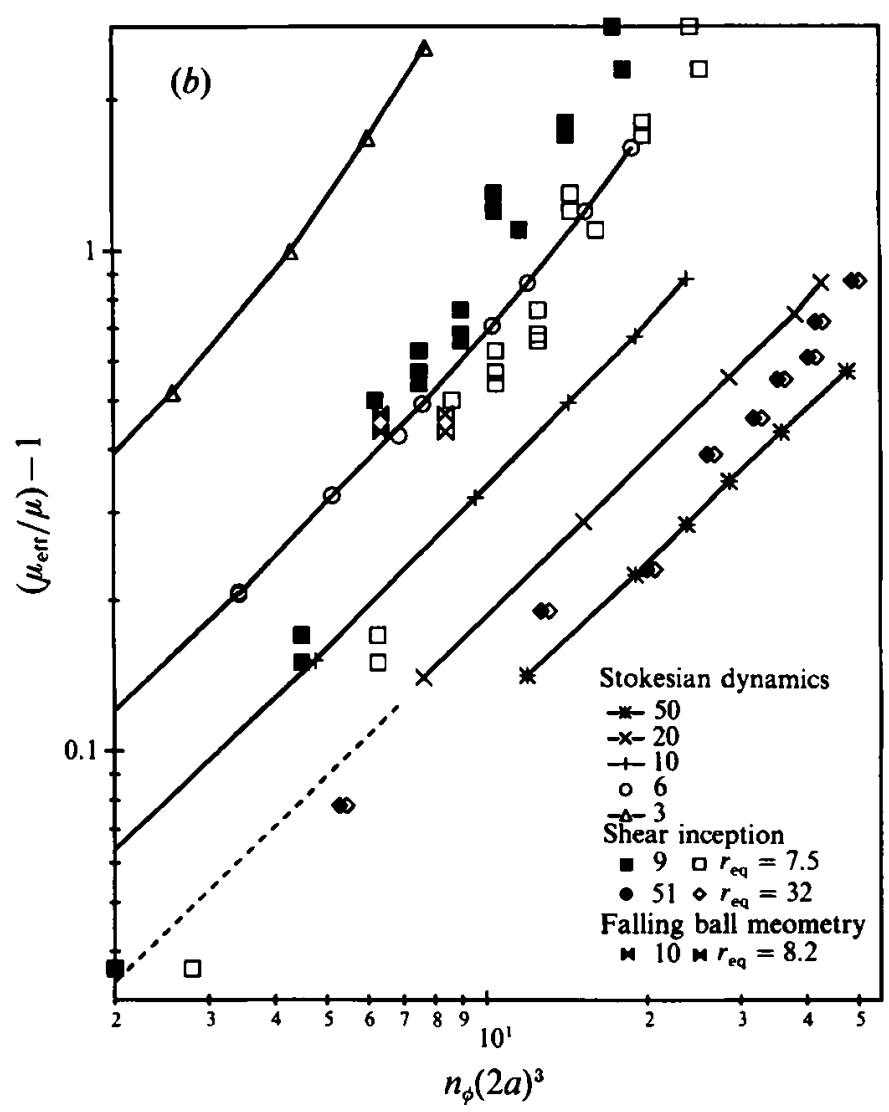

FIGURE 8. Double logarithmic plot of the viscosity of equilibrium dispersions of spheroids $v s$. volume fraction. Also included are the viscosity increments relative to pure solvent measured at the inception of shear by Bibbo et al. (1985) $\left(a: r_{p}=17\right.$ and 23) and by Bibbo (1987) $\left(b: r_{p}=9\right.$ and 51). Results of falling ball rheometry ( $a$ : Milliken et al. 1989; $b$ : Powell et al. 1990) are also shown. The open symbols correct for the cylindrical shape of the nylon rods used in the experiments.

that translational mixing alone accounts for a large fraction of the difference in the extensional viscosities of nematics and efc-lattices, possibly because of the disruption of the very regular columnar structures typical of the latter geometry. The partial misalignment of the spheroidal axes in the liquid crystalline phase however $\left(\left\langle P_{2}\right\rangle \approx 0.55\right.$ at $\left.\phi=0.33\right)$, is the dominant cause for the lower Trouton viscosity. Also included in figure 10 for illustrative purposes is the effect of lubrication interactions on the calculated viscosity. It is seen to be modest, increasing monotonically with density as expected.

Milliken et al. (1989) measured the effective viscosity of isotropic rod suspensions by falling ball rheometry. Unlike more traditional rheometric techniques, this method offers the advantage that the microstructure of the dispersion may not be significantly perturbed by the measurement, i.e. the orientation distribution of the rods may remain essentially at its initial state during the experiment (Powell et al. 1989). The effective viscosity is calculated from the sedimentation rate of a spherical probe through the dispersion. Data are reported up to a concentration of $5 \%$ in figure 7 for isotropic dispersions of polymethyl methacrylate (PMMA) rods of aspect ratio 19.83, and rayon fibres of aspect ratio 18.5. In concordance with our observations, Milliken et al. (1989) measured a linear increase of the effective viscosity with volume fraction up to 


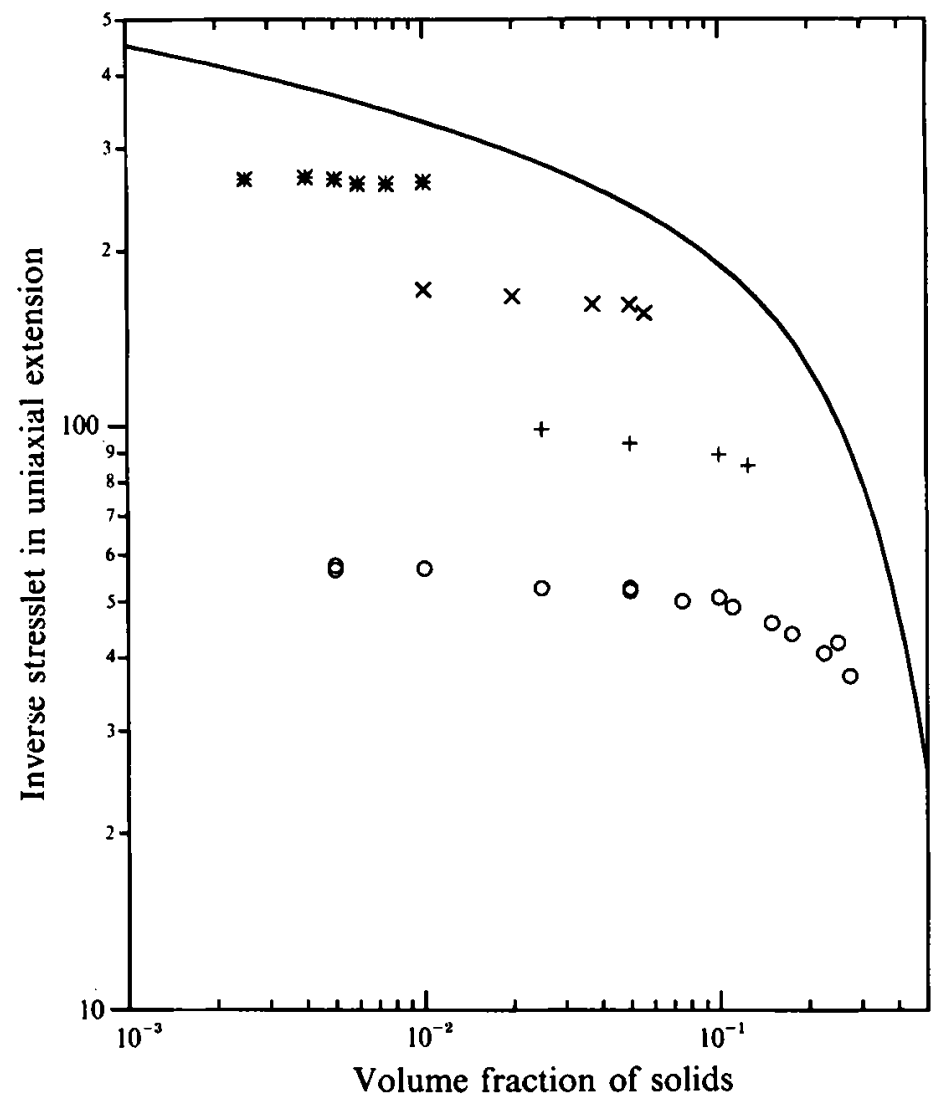

FIGURE 9. Transition from dilute to semi-dilute behaviour in isotropic dispersions. The stresslet in the principal direction of strain (normalized by $8 \pi a^{3} \mu E$, with $E$ the rate of uniaxial extension) gradually increases with volume fraction as the suspension deviates from the dilute limit. The symbols are as in the two previous figures. Also shown is the theoretical prediction due to Shaqfeh \& Fredrickson (1990).

concentrations much beyond the theoretical limit of dilute suspension behaviour $\left(\phi<r_{p}^{-2}\right)$. For cylindrical rods of aspect ratio 19.83 , they locate the transition to a nondilute response at $\phi=0.125$. Consistent with this finding, our simulations for suspensions of spheroids with $r_{p}=10,20$ and 50 predict very little deviation from linearity up to the highest concentrations studied. (The quality of the linear regressions is better than 0.999.) Moreover, a careful inspection of the data by Milliken et al. (1989) at low volume fractions reveals a slightly convex relation between $\mu_{\text {eff }}$ and $\phi$, in agreement with our results. Quantitatively however, our simulations appear to underestimate the effective viscosity by a factor of almost 2 , even though Milliken et al. (1989) claim a very good agreement with dilute suspension theory (Brenner 1974; Haber \& Brenner 1984). Since the particles used in the study were cylindrical, an equivalent spheroidal shape must be assumed to make the comparison meaningful. Conventionally, the effective aspect ratio is defined as the elongation of a spheroid with the same period of rotation in simple shear flow as the rod. Using the formula for slender blunt-ended bodies given by Brenner (1974), one easily finds $r_{\mathrm{eq}}=14.2$ for the PMMA sample, and $r_{\mathrm{eq}}=13.5$ for the rayon fibres. The particle dimension can also be adjusted to improve the fit. We determine the length of the major semi-axis by matching the stresslet of the cylindrical rods to that of the equivalent spheroids at infinite dilution (i.e. as $n_{\phi} \rightarrow 0$ ). By means of the material constants tabulated in 


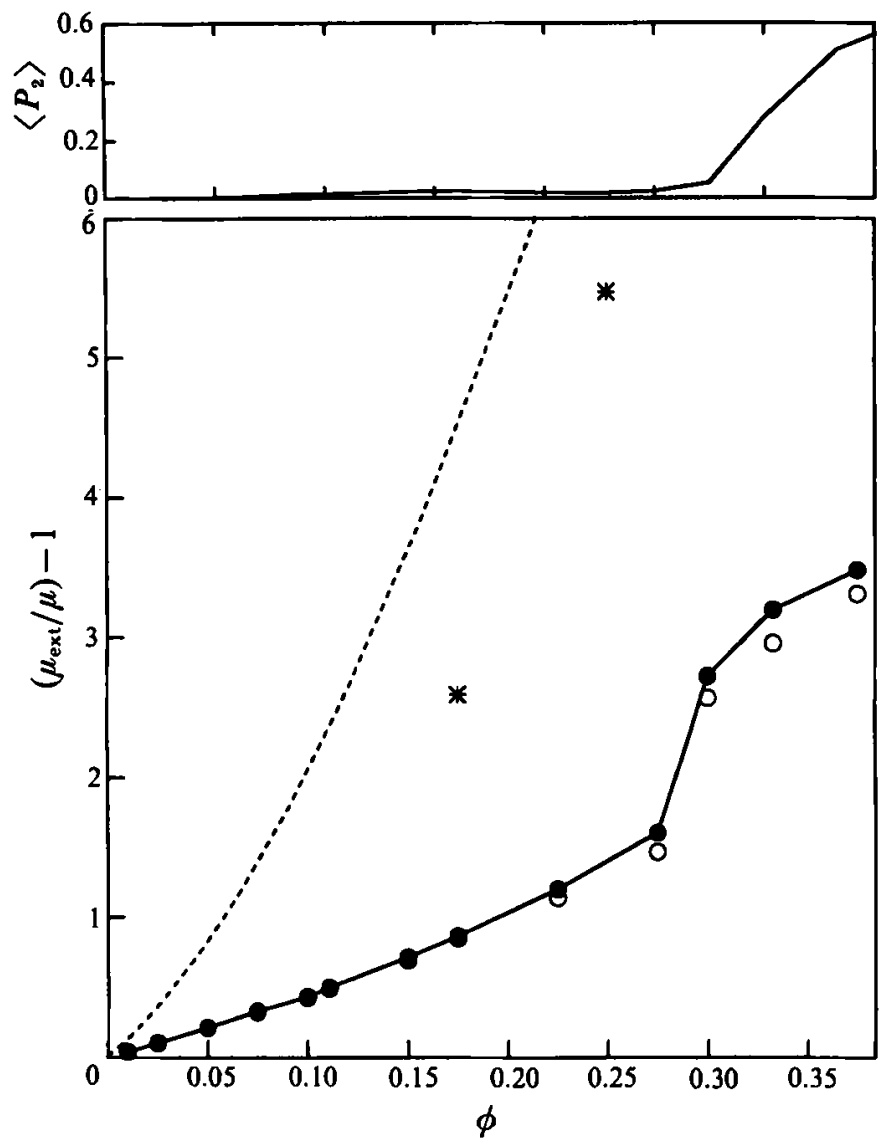

FIGURE 10. Concentration dependence of the extensional viscosity of equilibrium dispersions of spheroids of aspect ratio 6 . In the nematic region (characterized by a non-zero-order parameter, see top graph), the principal axis of strain lies along the director of the liquid crystal. The asterisks are for random microstructures of parallel ellipsoids. The dotted line shows data for efc-lattices (Claeys \& Brady 1993b). Lubrication interactions have been suppressed to give the open circles.

(Brenner (1974), one can calculate a small correction, $\left(a_{\mathrm{eq}} / a_{\mathrm{rod}}\right)^{3}=1.11$ for both test particles. As shown in figure 7, these refinements (which shift the concentration axis by a factor $\left.\frac{2}{3}\left(a_{\text {eq }} / a_{\text {rod }}\right)^{3}\left(r_{\text {rod }} / r_{\text {eq }}\right)^{2}\right)$ do not significantly reduce the discrepancies between simulation and experiment, since the revised data lie closer to the curve for $r_{p}=20$ than along $r_{\text {eq }}=14$. Even at the lowest volume fraction for which measurements were made, the agreement is unsatisfactory. How can this be reconciled with the fact that we equated the stresslets of the particles and of the equivalent spheroids at infinite dilution, and why do Milliken et al. (1989) find a much better agreement with theory (their figure 5) than we can offer?

Milliken $e t$ al. use the expression for the intrinsic viscosity $[\eta]=\left(\mu_{\mathrm{etr}} / \mu-1\right) \phi$ from the work by Haber \& Brenner (1984) on dilute monodisperse suspensions of centrosymmetric particles at small rotary Péclet number (i.e. under the assumption that the disorienting effect of Brownian motion overwhelms the tendency for alignment due to the imposed rate of strain):

$$
[\eta]=\frac{1}{3}\left(Q_{1}+Q_{2}+Q_{3}\right)+\left(q_{1}+q_{2}+q_{3}\right)-\frac{5}{6} \frac{N_{T}^{2}}{{ }^{r} K_{T}} .
$$


All the symbols on the right-hand side are purely geometric material constants which, for spheroids, are functions of the aspect ratio only (see Haber \& Brenner 1984 for their definition). In their derivation of (6.7), Haber \& Brenner (1984) emphasize that the last term arises from two contributions to the stress at steady state: (i) the mismatch between the angular velocity of the particle and the local vorticity of the flow, and (ii) the Brownian couple tending to restore the orientation distribution to a fully random state, annihilating the perturbation introduced by the straining field. For axisymmetric bodies, in the limit of strong Brownian motion, these two effects cancel each other identically $\left(N_{T}=0\right)$. This indicates that they are of comparable magnitude, and hence that it is inconsistent (at steady state and for small Péclet numbers) to neglect one and retain the other. In essence, if a rod is easily rotated by the flow (and therefore alleviates the stress by 'yielding' to the straining field), its motion will create an anisotropic orientation distribution which, in turn, generates a Brownian stress. In our simulations, only the hydrodynamic contributions to the stress are evaluated; the Brownian part is not. $†$ However the latter is exactly zero for the isotropic microstructures considered here, and the entire stress in the dispersion is hydrodynamic (in the absence of interparticle forces). Bear in mind that these configurations were obtained by a MonteCarlo technique, and that their orientation distribution is not a solution of the evolution equation in the presence of an imposed flow, in contrast to the underlying assumption of steady state in (6.7). Alternatively, we calculate the properties of isotropic suspensions of non-Brownian rods, i.e. in the limit of infinite Péclet number, for which (6.7) does not hold. Indeed, our simulation results are in very good agreement with dilute suspension theory, provided that one recognizes that all orientations are equally probable, identically, and hence that the Brownian contribution to the viscosity is zero. Equation (6.7) then becomes

$$
[\eta]=\frac{1}{3}\left(Q_{1}+Q_{2}+Q_{3}\right)+\left(q_{1}+q_{2}+q_{3}\right)-\frac{5}{6}\left(\frac{N_{1}^{2}}{{ }^{r} K_{1}}+\frac{N_{2}^{2}}{{ }^{r} K_{2}}+\frac{N_{3}^{2}}{{ }^{r} K_{3}}\right),
$$

as first calculated by Batchelor (1970a) for dilute suspensions of triaxial ellipsoids (see also Haber \& Brenner 1984, their equation (8.8)). (One can also use Brenner's equation (4.25) (Brenner 1974), since it does not assume steady state and is correct for any orientation distribution.) The excellent fit of the data of Milliken et al. (1989) by equation (6.7) is misleading because it suggests that their experiments measure both the hydrodynamic and the Brownian stress in the suspension, even though the rotary Péclet number $P e \gg 1.9 \times 10^{9}$ for the centimer-sized rods which they use. The authors recognized this and state that 'the effect of Brownian forces was negligible.' Yet they rely on (6.7) for comparison with theory, arguing erroneously that this formula should apply for random dispersions of axisymmetric particles, whether this randomness is caused by Brownian motion or by 'whatever means' (Powell 1991). This is untrue, and the excellent agreement noted by Milliken et al. (1989) must be called fortuitous. Powell et al. (1989) report data for millimetre-sized rods of aspect ratio 10 using falling ball rheometry, and find that (6.7) overestimates the viscosity, in accord with our expectations. Although the authors blame the deviation on the use of slender-body theory in the derivation of (6.7), it is clear that the Brownian contribution to the stress, which is included in (6.7), should be negligible in their experimental system too. As

$\dagger$ The expression for the Brownian stress involves the divergence of a combination of $N$-particle resistance tensors, $\boldsymbol{\nabla} \cdot\left[\boldsymbol{R}_{s U} \cdot \boldsymbol{R}_{F V}^{-1}\right]$ (Brady \& Bossis 1988; Bossis \& Brady 1989), and its evaluation requires a full dynamic simulation. This problem, and other complications associated with thermal motion, form a subject for further study. 


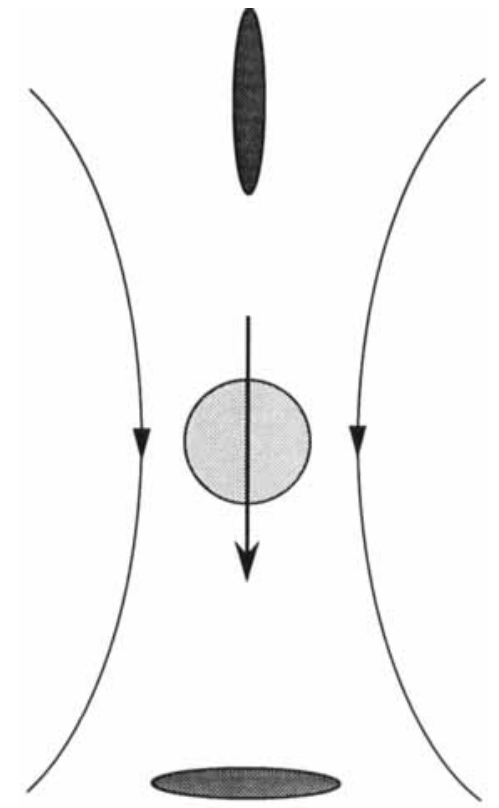

FIGURE 11. Influence of the velocity field caused by a sedimenting sphere on the microstructure of a suspension of force-free rods. This sketch illustrates the partial order induced in a dispersion of spheroids by the extensional components of the disturbance velocity. The spheroids are drawn in their preferred orientation (i.e. along the local principal axis of extension).

shown in figure 7 , however, their viscosity measurements (performed at $\phi=0.05$ only) also yielded values slightly higher than those predicted by our simulations on equilibrium hard-rod dispersions. (The equivalent aspect ratio of their cylindrical particles is 8.2.)

A possible explanation for the discrepancy between the experimental results and the (correct) theory assumes that the perturbation, however slight, of the original microstructure by the falling ball contributes substantially to the measured viscosity. As shown in figure 10, an alignment of the particles, even if incomplete, can cause a sizeable increase in the extensional resistance of the dispersion, i.e. in $\eta_{T}$. Since this component of the stresslet dominates the effective viscosity for sufficiently slender bodies (vide supra), a partial ordering of the rods during the measurement, as suggested by simulations using boundary elements methods $\dagger$ (Phan-Thien \& Graham 1991; Phan-Thien, Zheng \& Graham 1991), may lead to an overestimation of $[\eta]$. As sketched in figure 11 , the velocity disturbance generated by the falling sphere (which acts as a point force to a first approximation) is expected to bring about this partial orientational order in the dispersion. Additionally, the mechanism that lead Koch \& Shaqfeh (1989) to propose that sedimenting suspensions of spheroids are unstable, could cause an increase of the particle density in the vicinity of the falling ball. In essence, the rods immediately above the sphere experience a straining field which aligns them in the main direction of the flow, so that they are carried along by the fluid more readily than the fibres below the sphere, which are oriented perpendicular to gravity by the velocity field

$\dagger$ Although the constitutive equation assumed by Phan-Thien \& Graham (1991) for the fibre suspension is somewhat ad hoc, their simulation should capture qualitative features such as flowinduced alignment. 
(figure 11). This results in a crowding of the particles near the sedimenting ball, with a concomitant increase in its drag (and therefore in the apparent viscosity). Milliken et al. (1989) have shown, however, that the dependence of the sedimentation rate on the diameter of the container can be factored out by using Faxén's correction, derived for a Newtonian fluid (Bohlin 1960). Hence, they argued, the suspension can be characterized by a scalar viscosity, and its microstructure must be isotropic. It is not clear whether the inducement of a preferred rod orientation by the falling ball would suffice to explain the magnitude of the discrepancy between the measurements and our data, nor why Faxén's correction would then apply, but it seems the most plausible hypothesis. Mondy et al. (1990) performed falling ball rheometry on aligned fibre dispersions, and observed that the sphere sediments faster than in random samples. This does not contradict our argument, since the induced orientation distribution that we propose as an explanation for the deviation of the simulation results from the experiments of Milliken et al. (1989) is not uniform. Our study on crystals of parallel spheroids (Claeys \& Brady $1993 \mathrm{~b}$ ) demonstrates that the apparent viscosity of aligned dispersions can be higher or lower than that of isotropic structures, depending on the orientation of the fibres relative to the principal axes of extension of the flow. We did not attempt to reproduce Mondy et al.'s (1990) results numerically, because it is unclear which components of the stress response, which is anisotropic for suspensions of aligned rods (Claeys \& Brady 1993b), are accessed by falling ball rheometry.

Transient measurements of the viscosity, which probe the dispersion while it is still fully isotropic, circumvent the concerns about induced microstructure raised for steady-state techniques such as falling ball rheometry. Bibbo, Dinh \& Armstrong (1985) evaluated the suspension viscosity for nylon fibres of aspect ratio 16.7 and 22.7 $\left(r_{\text {eq }}=12.3\right.$ and 15.9 respectively) at the inception of shear flow. Their data are superposed on the simulation results in figure 8(a). Also shown are the values after adjustment for the cylindrical shape of the particles (performed as above). Although the scatter in the measurements is too large to support our numerical predictions conclusively, the agreement is reasonable: while the experimental results and the calculated viscosities sometimes differ by a factor of two, most of the points reported by Bibbo et al. (1985) fall between the curves for $r_{p}=10$ and 20, as expected in view of the equivalent aspect ratio. The reliability of the empirical data for the most slender particles, however, is somewhat questionable due to the small gap width of the rheometer compared to the length of the $5 \mathrm{~mm}$ fibres (Powell 1991). For comparison, the viscosities measured by falling ball rheometry (Milliken et al. 1989) are also included in figure 8(a). These results lie within the spread on the data of Bibbo et al. (1985), but give values systematically higher than the simulations. Viscosities extrapolated to zero strain for dispersions of nylon fibres of aspect ratio $9\left(r_{\mathrm{eq}}=7.5\right)$ and $51\left(r_{\text {eq }}=32\right)$ (Bibbo 1987) also agree quite satisfactorily with the predictions (figure $8 b$ ), although the data for the blunter cylinders hint at a steeper dependence on concentration than the one found numerically.

\section{Conclusions}

The methodology of 'Stokesian dynamics', a hydrodynamically accurate simulation technique for dispersions of spheres, has been extended to spheroidal particles. Absolutely convergent expressions for the motion of the solid phase can be obtained by applying O'Brien's (1979) renormalization scheme. The Ewald summation technique (Beenakker 1986; Brady et al. 1988) speeds up the evaluation of the lattice sums introduced by the periodic boundary conditions used to construct unbounded systems. 
A truncation of the stress moment expansion after the first two multipoles, keeping only the effect of the force, torque and stresslet, and hence requiring only 11 unknowns per particle, is adequate, as illustrated in $\S 5$, where we compared the extensional viscosity of a dispersion of spheroids to that of a suspension of beads rigidly connected into strings. The hydrodynamic transport properties of equilibrium hard-ellipsoid structures were examined in the last section. A nearly linear relation between the effective viscosity of isotropic dispersions and the volume fraction of fibres is observed over a range of densities extending much beyond the limits of the dilute concentration regime, as first quantitatively shown by falling ball rheometry (Milliken et al. 1989). The calculations are also in good agreement with measurements of the stress in a parallel-plate rheometer at the inception of shear (Bibbo et al. 1985; Bibbo 1987). The microstructural change accompanying the thermodynamic isotropic-to-nematic transition dramatically influences the rheological properties of rod dispersions (figure 10). A study into the hydrodynamic transport properties of model configurations possessing long-ranged orientational order was therefore undertaken and forms the subject of a companion paper (Claeys \& Brady 1993b).

In practice, the impact of the imposed flow on the microstructure of the dispersion is as important as the influence of the dispersion topology on its properties. Controlling the flow of fibre suspensions thus requires insight into both facets of the problem. Dynamic simulations, investigating the occurrence of flow-induced alignment for instance, therefore form a topic of future research. The inclusion of Brownian effects is also a subject to be addressed.

Appendix A. Correspondence of the non-local singularity representation of the disturbance velocity due to a spheroid in a straining field with slenderbody theory

The singularity representation for the fluid flow disturbance due to an isolated stationary ellipsoid immersed in a purely extensional flow characterized by the rate-ofstrain tensor $\boldsymbol{E}$ infinitely far away from the particle, is (Kim 1985)

$$
\begin{aligned}
& u(x)-E \cdot x=-\frac{1}{2} \alpha_{1} E:\left[d_{p} d_{p} \wedge \int_{-c_{p}}^{c_{p}}\left(c_{p}^{2}-\xi_{p}^{2}\right) \nabla \wedge J\left(x-\xi_{p}\right) \mathrm{d} \xi_{p}\right] \\
& \quad+E: K_{S E}^{p}: \int_{-c_{p}}^{c_{p}}\left(c_{p}^{2}-\xi_{p}^{2}\right)\left\{1+\left(c_{p}^{2}-\xi_{p}^{2}\right) \frac{1-e_{p}^{2}}{8 e_{p}^{2}} \nabla^{2}\right\} \frac{1}{2}\left(\nabla+{ }^{\mathrm{T}} \nabla\right) J\left(x-\xi_{p}\right) \mathrm{d} \xi_{p} .
\end{aligned}
$$

The shape specific tetradic $K_{S E}^{p}$ and the coefficient $\alpha_{1}$ are known for spheroids (Chwang $\& \mathrm{Wu} 1975 ; \mathrm{Kim} 1985)$. We decompose $\nabla=d_{p}\left(d_{p} \cdot \nabla\right)+\nabla_{\perp}=d_{p} \partial / \partial \xi_{p}+\nabla_{\perp}$ and integrate by parts:

$$
\begin{aligned}
\boldsymbol{u}= & E \cdot \boldsymbol{x}+\boldsymbol{E}: \boldsymbol{K}_{S E}^{p}: \int_{-c_{p}}^{c_{p}} \xi_{p}\left\{1+\left(c_{p}^{2}-\xi_{p}^{2}\right) \frac{1-e_{p}^{2}}{4 e_{p}^{2}} \nabla^{2}\right\} \frac{1}{2}\left(d_{p} J+{ }^{\mathrm{T}} d_{p} J\right) \mathrm{d} \xi_{p} \\
& +E: K_{S E}^{p}: \int_{-c_{p}}^{c_{p}}\left(c_{p}^{2}-\xi_{p}^{2}\right)\left\{1+\left(c_{p}^{2}-\xi_{p}^{2}\right) \frac{1-e_{p}^{2}}{8 e_{p}^{2}} \nabla^{2}\right\} \frac{1}{2}\left(\nabla_{\perp}+{ }^{\mathrm{T}} \nabla_{\perp}\right) J \mathrm{~d} \xi_{p} \\
& -\frac{1}{2} \alpha_{1} E:\left[d_{p} d_{p} \wedge \int_{-c_{p}}^{c_{p}}\left(c_{p}^{2}-\xi_{p}^{2}\right) \nabla_{\perp} \wedge J \mathrm{~d} \xi_{p}\right] .
\end{aligned}
$$


In the limit $e_{p} \rightarrow 1$, the first integral in (A 2) equates the effect of the spheroid with that of a linear distribution of Stokeslets along the symmetry axis, as predicted by slenderbody theory.

\section{REFERENCES}

AdLer, P. M. 1985 J. Theor. Appl. Mech., Special volume, pp. 73-100.

Allen, M. P. 1990 Phys. Rev. Lett. 65, 2881-2884.

Allen, M. P., Frenkel, D. \& Talbot, J. 1989 Comput. Phys. Rep. 9, 301-353.

Batchelor, G. K. 1970 a J. Fluid Mech. 41, 545-570.

BatChelor, G. K. $1970 b$ J. Fluid Mech. 44, 419-440.

BATCHeloR, G. K. 1971 J. Fluid Mech. 46, 813-829.

BAtCheloR, G. K. 1972 J. Fluid Mech. 52, 245-268.

BatChelor, G. K. 1976 J. Fluid Mech. 74, 1-29.

BAtChelor, G. K. \& GreEN, J. T. 1972 J. Fluid Mech. 56, 401-427.

Beenak Ker, C. W. J. 1986 J. Chem. Phys. 85, 1581-1582.

BibBo, M. A. 1987 Rheology of semiconcentrated fiber suspensions. PhD thesis, Massachusetts Institute of Technology.

Bibio, M. A., Dinh, S. M. \& Armstrong, R. C. 1985 J. Rheol. 29, 905-929.

Bohlin, T. 1960 Trans. R. Inst. Tech. (Stockholm) 155, 1-63.

BonneCAZE, R. T. 1991 Macroscopic properties of electrically interacting suspensions. PhD thesis, California Institute of Technology.

Bossis, G. \& Brady, J. F. 1989 J. Chem. Phys. 91, 1866-1874.

Brady, J. F. \& Bossis, G. 1988 Ann. Rev. Fluid Mech. 20, 111-157.

Brady, J. F., Phillips, R. J., Lester, J. C. \& Bossis, G. 1988 J. Fluid Mech. 195, 257-280.

BrenNer, H. 1964 Chem. Engng Sci. 19, 631-651.

BrenNer, H. 1966 Chem. Engng Sci. 21, 97-109.

BRENNER, H. 1974 Intl J. Multiphase Flow 1, 195-341.

Chwang, A. T. \& Wu, T. Y.-T. 1974 J. Fluid Mech. 63, 607-622.

Chwang, A. T. \& Wu, T. Y.-T. 1975 J. Fluid Mech. 67, 787-815.

ClaEYs, I. L. 1991 Hydrodynamic transport properties of suspensions of non-Brownian prolate spheroids. PhD thesis, California Institute of Technology.

Claeys, I. L. \& Brady, J. F. 1989 PhysicoChem. Hydrodyn. 11, 261-293.

Claeys, I. L. \& Brady, J. F. 1993 a J. Fluid Mech. 251, 411-442.

Claeys, I. L. \& Brady, J. F. $1993 b$ J. Fluid Mech. 251, 479-500.

CUCKIER, R. I. 1983 Macromol. 17, 252-255.

Dol, M. \& Edwards, S. F. 1986 The Theory of Polymer Dynamics. Clarendon.

Durlofsky, L. \& Brady, J. F. 1987 Phys. Fluids 30, 3329-3341.

Durlofsky, L., Brady, J. F. \& Bossis, G. 1987 J. Fluid Mech. 180, 21-49.

Ewald, P. P. 1921 Ann. Phys. 64, 253-287.

Frenkel, D. \& Mulder, B. M. 1985 Mol. Phys. 55, 1171-1192.

Frenkel, D., Mulder, B. M. \& MCTague, J. P. 1984 Phys. Rev. Lett. 52, 287-290.

Gennes, P. G. DE 1971 J. Chem. Phys. 55, 572-579.

Glendinning, A. B. \& Russel, W. B. 1982 J. Colloid Interface Sci. 89, 124-143.

Haber, S. \& BRENNER, H. 1984 J. Colloid Interface Sci. 97, 496-514.

HAPPEL, J. \& BRENNER H. 1973 Low Reynolds Number Hydrodynamics. Martinus Nijhoff.

Hasimoto, H. 1959 J. Fluid Mech. 5, 317-328.

HinCH, E. J. 1977 J. Fluid Mech. 83, 695-720.

Jeffrey, D. J. 1973 Proc. R. Soc. Lond. A 335, 355-367.

KIM, S. 1985 Intl J. Multiphase Flow 5, 713-719.

KIM, S. 1986 Intl J. Multiphase Flow 12, 469-491.

KiM, S. \& Arunachalam, P. V. 1987 J. Fluid Mech. 178, 535-547. 
Koch, D. L. \& ShaQfeH, E. S. G. 1989 J. Fluid Mech. 209, 521-542.

LADD, A. J. C. 1990 J. Chem. Phys. 93, 3484-3494.

LADYZHENSKayA, O. A. 1963 The Mathematical Theory of Viscous Incompressible Flow. Gordon \& Breach.

Megen, W. van \& Underwood, S. M. 1989 J. Chem. Phys. 91, 552-559.

Metropolis, N., Rosenbluth, A. W., Rosenbluth, M. N., Teller, A. H. \& Teller, E. 1953 J. Chem. Phys. 21, 1087-1092.

Mewis, J. \& Metzner, A. B. 1974 J. Fluid Mech. 62, 593-600.

Milliken, W. J., Gottlieb, M., Graham, A. L., Mondy, L. A. \& Powell, R. L. 1989 J. Fluid Mech. 202, 217-232.

Mondy, L. A., Morrison, T. G., Graham, A. L. \& Powell, R. L. 1990 Intl J. Multiphase Flow 16, $651-662$.

NiJBoer, B. R. A. \& WeTte, F. F. DE 1957 Physica 23, 309-321.

O'Brien, R. W. 1979 J. Fluid Mech. 91, 17-39.

Ottewill, R. H. \& Williams, N. ST. J. 1987 Nature 325, 232-234.

Perram, J. W. \& Wertheim, M. S. 1985 J. Comput. Phys. 58, 409-416.

Perry, P. \& Throop, G. J. 1972 J. Chem. Phys. 57, 1827-1829.

Phan-Thien, N. \& Graham, A. L. 1991 Rheol. Acta 30, 44-57.

Phan-Thien, N., Zheng, R. \& Graham, A. L. 1991 J. Statist. Phys. 62, 1173-1195.

Phillies, G. D. J. 1987 Macromol. 20, 558-564.

Phillips, R. J., Brady, J. F. \& Bossis, G. 1988 a Phys. Fluids 31, 3462-3472.

Phillips, R. J., Brady, J. F. \& Bossis, G. 1988 b Phys. Fluids 31, 3473-3479.

Pittman, J. F. T. \& Bayram, J. 1990 Intl J. Multiphase Flow 16, 545-559.

Powell, R. L. 1991 J. Statist. Phys. 62, 1073-1094.

Powell, R. L., Mondy, L. A., Stoker, G. G., Milliken, W. J. \& Graham, A. L. 1989 J. Rheol. 33, $1173-1188$.

Pusey, P. N. \& Megen, W. van 1983 J. Chem. Phys. 44, 285-291.

Revay, J. M. \& Higdon, J. J. L. 1992 J. Fluid Mech. 243, 15-32.

SeE, H., Dol, M. \& LaRSON, R. 1990 J. Chem. Phys. 92, 792-800.

SHAQFeh, E. S. G. 1988 Phys. Fluids 31, 2405-2425.

Shaqfeh, E. S. G. \& Fredrickson, G. H. 1990 Phys. Fluids A 2, 7-24.

SMith, W. R. \& Henderson, D. 1970 Molec. Phys. 19, 411-415.

SPIElman, L. \& GoRen, S. L. 1968 Environ. Sci. Tech. 2, 279-287.

Thirumalal, D. 1986 J. Chem. Phys. 84, 5869-5873.

Werff, J. C. van Der, Kruiff, C. G. De, Blom, C. \& Mellema, J. 1989 Phys. Rev. A 39, $795-807$.

Verlet, L. \& Weiss, J. J. 1972 Phys. Rev. A 5, 939-952.

Vieillard-Baron, J. $1970 \mathrm{~J}$. Chem. Phys. 56, 4729-4744.

Weinbaum, S., Ganatos, P. \& Yan, Z. Y. 1990 Ann. Rev. Fluid Mech. 22, 275-316.

Wheeler, L. M., Lodge, T. P., Hanley, B. \& Tirrell, M. 1987 Macromol. 20, 1120-1129.

White, M. L. \& Dorion, G. H. 1961 J. Polymer Sci. 55, 731-740.

Zuzovsky, M., Adler, P. M. \& Brenner, H. 1983 Phys. Fluids 26, 1714-1723. 